Louisiana State University

LSU Digital Commons

Faculty Publications

Department of Physics \& Astronomy

10-10-2016

\title{
THREE TEMPERATE NEPTUNES ORBITING NEARBY STARS
}

\author{
Benjamin J. Fulton \\ University Hawaii Institute for Astronomy \\ Andrew W. Howard \\ University Hawaii Institute for Astronomy \\ Lauren M. Weiss \\ University of California, Berkeley \\ Evan Sinukoff \\ University Hawaii Institute for Astronomy \\ Erik A. Petigura \\ California Institute of Technology
}

See next page for additional authors

Follow this and additional works at: https://digitalcommons.Isu.edu/physics_astronomy_pubs

\section{Recommended Citation}

Fulton, B., Howard, A., Weiss, L., Sinukoff, E., Petigura, E., Isaacson, H., Hirsch, L., Marcy, G., Henry, G., Grunblatt, S., Huber, D., Braun, K., Boyajian, T., Kane, S., Wittrock, J., Horch, E., Ciardi, D., Howell, S., Wright, J., \& Ford, E. (2016). THREE TEMPERATE NEPTUNES ORBITING NEARBY STARS. Astrophysical Journal, 830 (1) https://doi.org/10.3847/0004-637X/830/1/46

This Article is brought to you for free and open access by the Department of Physics \& Astronomy at LSU Digital Commons. It has been accepted for inclusion in Faculty Publications by an authorized administrator of LSU Digital Commons. For more information, please contact ir@lsu.edu. 


\section{Authors}

Benjamin J. Fulton, Andrew W. Howard, Lauren M. Weiss, Evan Sinukoff, Erik A. Petigura, Howard Isaacson, Lea Hirsch, Geoffrey W. Marcy, Gregory W. Henry, Samuel K. Grunblatt, Daniel Huber, Kaspar Von Braun, Tabetha S. Boyajian, Stephen R. Kane, Justin Wittrock, Elliott P. Horch, David R. Ciardi, Steve B. Howell, Jason T. Wright, and Eric B. Ford 


\title{
THREE TEMPERATE NEPTUNES ORBITING NEARBY STARS*
}

\author{
Benjamin J. Fulton ${ }^{1,16}$, Andrew W. Howard ${ }^{1}$, Lauren M. Weiss ${ }^{2,17}$, Evan Sinukoff ${ }^{1,18}$, Erik A. Petigura ${ }^{3,19}$, \\ Howard IsaACSON $^{2}$, Lea Hirsch ${ }^{2}$, Geoffrey W. Marcy ${ }^{2}$, Gregory W. Henry ${ }^{4}$, Samuel K. Grunblatt ${ }^{1}$, Daniel Huber ${ }^{5,6,7}$, \\ Kaspar von Braun ${ }^{8}$, Tabetha S. Boyajian ${ }^{9}$, Stephen R. Kane ${ }^{10}$, Justin Wittrock ${ }^{10}$, Elliott P. Horch ${ }^{11}$, \\ David R. Ciardi ${ }^{12}$, Steve B. Howell ${ }^{13}$, Jason T. Wright ${ }^{14,15}$, And Eric B. Ford ${ }^{14,15}$ \\ ${ }^{1}$ Institute for Astronomy, University of Hawai i, 2680 Woodlawn Drive, Honolulu, HI 96822, USA; bfulton@ hawaii.edu \\ ${ }^{2}$ Department of Astronomy, University of California, Berkeley, CA 94720, USA \\ ${ }^{3}$ California Institute of Technology, Pasadena, California, USA \\ ${ }^{4}$ Center of Excellence in Information Systems, Tennessee State University, 3500 John A. Merritt Blvd., Box 9501, Nashville, TN 37209, USA \\ ${ }^{5}$ SETI Institute, 189 Bernardo Avenue, Mountain View, CA 94043, USA \\ ${ }^{6}$ Sydney Institute for Astronomy (SIfA), School of Physics, University of Sydney, NSW 2006, Australia \\ ${ }^{7}$ Stellar Astrophysics Centre, Department of Physics and Astronomy, Aarhus University, Ny Munkegade 120, DK-8000 Aarhus C, Denmark \\ ${ }^{8}$ Lowell Observatory, Flagstaff, AZ 86001, USA \\ ${ }^{9}$ Yale University, New Haven, CT 06520, USA \\ ${ }^{10}$ Department of Physics and Astronomy, San Francisco State University, 1600 Holloway Avenue, San Francisco, CA 94132, USA \\ ${ }^{11}$ Department of Physics, Southern Connecticut State University, New Haven, CT 06515, USA \\ ${ }^{12}$ NASA Exoplanet Science Institute, Caltech, MS 100-22, 770 South Wilson Avenue, Pasadena, CA 91125, USA \\ ${ }^{13}$ NASA Ames Research Center, Moffett Field, CA 94035, USA \\ ${ }_{15}^{14}$ Center for Exoplanets and Habitable Worlds, The Pennsylvania State University, University Park, PA 16802, USA \\ ${ }^{15}$ Department of Astronomy \& Astrophysics, The Pennsylvania State University, University Park, PA 16802, USA \\ Received 2015 December 31; revised 2016 June 29; accepted 2016 July 1; published 2016 October 10
}

\begin{abstract}
We present the discovery of three modestly irradiated, roughly Neptune-mass planets orbiting three nearby Solartype stars. HD $42618 \mathrm{~b}$ has a minimum mass of $15.4 \pm 2.4 M_{\oplus}$, a semimajor axis of 0.55 au, an equilibrium temperature of $337 \mathrm{~K}$, and is the first planet discovered to orbit the solar analogue host star, HD 42618. We also discover new planets orbiting the known exoplanet host stars HD 164922 and HD $143761(\rho \mathrm{CrB})$. The new planet orbiting HD 164922 has a minimum mass of $12.9 \pm 1.6 M_{\oplus}$ and orbits interior to the previously known Jovian mass planet orbiting at $2.1 \mathrm{au}$. HD $164922 \mathrm{c}$ has a semimajor axis of 0.34 au and an equilibrium temperature of 418 K. HD $143761 \mathrm{c}$ orbits with a semimajor axis of $0.44 \mathrm{au}$, has a minimum mass of $25 \pm 2 M_{\oplus}$, and is the warmest of the three new planets with an equilibrium temperature of $445 \mathrm{~K}$. It orbits exterior to the previously known warm Jupiter in the system. A transit search using space-based CoRoT data and ground-based photometry from the Automated Photometric Telescopes (APTs) at Fairborn Observatory failed to detect any transits, but the precise, high-cadence APT photometry helped to disentangle planetary-reflex motion from stellar activity. These planets were discovered as part of an ongoing radial velocity survey of bright, nearby, chromospherically inactive stars using the Automated Planet Finder (APF) telescope at Lick Observatory. The high-cadence APF data combined with nearly two decades of radial velocity data from Keck Observatory and gives unprecedented sensitivity to both short-period low-mass, and long-period intermediate-mass planets.
\end{abstract}

Key words: planetary systems - stars: individual (HD 42618, HD 164922, HD 143761)

Supporting material: machine-readable table

\section{INTRODUCTION}

The mass function of extrasolar planets potentially offers rich clues to the processes that shape their growth and evolution. This mass function is known to rise with decreasing planet mass based on the discovery and characterization of planets orbiting nearby stars (Howard et al. 2010; Mayor et al. 2011). Although the Kepler mission discovered thousands of transiting exoplanets allowing for detailed characterization of the planet radius distribution (Howard et al. 2012; Fressin et al. 2013; Petigura et al. 2013a), we have a much sparser

\footnotetext{
* Based on observations obtained at the W. M. Keck Observatory, which is operated jointly by the University of California and the California Institute of Technology. Keck time was granted for this project by the University of Hawai ' $i$, the University of California, and NASA.

16 National Science Foundation Graduate Research Fellow.

17 Ken and Gloria Levy Graduate Student Research Fellow.

18 Natural Sciences and Engineering Research Council of Canada Graduate Student Fellow.

${ }^{19}$ Hubble Fellow.
}

sample of planets orbiting nearby stars. We only know of 17 confirmed planets with measured minimum masses $M \sin i<30 M_{\oplus}$ and orbital periods $P>75$ days. $^{20}$ These low-mass, temperate planets reside in an region of parameter space that must be explored in order to understand the formation of the extremely abundant population of close-in super-Earths.

The discovery that the occurrence rate of Jovian planets increases at orbital distances of 1-3 au (Cumming et al. 2008) has been suggested to be a sign that the ice line is important to the formation of Jovian planets. The increased abundance of solids in the protoplanetary disk beyond the ice-line is expected to speed up the coagulation of planetesimals and $\sim 10 M_{\oplus}$ cores that can undergo runaway gas accretion before the gas in the disk is dissipated (Ida \& Lin 2008).

The formation of close-in intermediate mass planets known as super-Earths or mini-Neptunes presents some challenges for

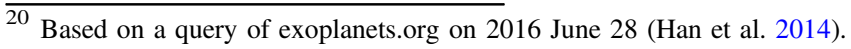


planet formation theory. It was initially thought that such planets should either remain small terrestrial planets, or if they grow to large cores then they should quickly accrete substantial nebular gas and grow to be gas giants (Ida \& Lin 2004; Mordasini et al. 2009). Alternatively, planets that form in situ likely require either a protoplanetary disk that is much more massive than the minimum mass solar nebula (Hansen \& Murray 2012; Chiang \& Laughlin 2013), an extremely metalrich disk, or fine tuning of the formation timescales (Lee et al. 2014). New models featuring gas-drag driven "pebble accretion" which offers a mechanism to transport solids in the disc (Chatterjee \& Tan 2014), or the delayed formation of super-Earth cores until the gas disk begins to dissipate (Lee \& Chiang 2015), could facilitate in situ formation of super-Earths and/or mini Neptunes.

Alternatively, migration from further out in the disk where there is plenty of material to form massive planet cores or super-Earths could explain the presence of these planets near their host stars. We would expect to see multi-planet systems in resonant chains if a slow, smooth migration were the dominant mechanism and this is not observed (Veras \& Ford 2012; Fabrycky et al. 2014). Another indication that migration is at play would be an increased occurrence rate of super-Earths at large orbital separations. The occurrence rates of super-Earths as a function of orbital period appears fairly flat to periods as long as 200 days (Petigura et al. 2013a), however the sensitivity to long-period planets from transit surveys is very low. In addition, the planet radius distribution can be influenced by a variety of different factors including stellar irradiation, and thermal evolution (Lopez 2014). Only a very small percentage by mass of volatiles can significantly inflate a planet's radius and hide the fundamental properties of the planet that encode information about the formation mechanism (Lopez \& Fortney 2014). RV surveys are now starting to discover a statistically useful sample of super-Earth to Neptune mass planets at larger orbital separations and lower stellar irradiance that will help to map out the details of the mass function for long-period super-Earth to Neptune mass planets.

The Eta-Earth RV survey of nearby stars (Howard et al. 2010) was conducted using the HIRES spectrograph at Keck observatory (Vogt et al. 1994). They searched for planets in a volume-limited sample of 166 nearby $G$ and $K$ dwarfs. With the catalog of planets detected in this survey (Howard et al. 2009, 2011a, 2011b, 2014), and the completeness limits calculated for each star they were able to measure the occurrence rate of small, short-period planets and show that planets with masses of 3-30 $M_{\oplus}$ are much more common than planets larger than $30 M_{\oplus}$. Although these low-mass planets are common, they are still very difficult to detect, requiring $>100$ measurements per star with $\lesssim 2 \mathrm{~m} \mathrm{~s}^{-1}$ precision. The number of nearby stars for which large, high-precision radial velocity datasets exist is increasing thanks to the proliferation of dedicated and robotic radial velocity facilities such as the APF and MINERVA (Swift et al. 2015) and the ongoing long-term surveys from Keck, HARPS-N (Motalebi et al. 2015), and HARPS (Pepe et al. 2004).

We are currently using the Automated Planet Finder (APF) telescope to conduct a RV survey of 51 of the brightest, and least chromospherically active, stars from the Eta-Earth survey. We capitalize on the robotic nature of the telescope to monitor the stars at high cadence for the entire four year duration of the survey. This survey builds on the Eta-Earth Survey, but with improved Doppler precision due to the high observing cadence and larger number of measurements. We will measure the occurrence rate and mass function of small planets in our local neighborhood using the new planets discovered by the APF-50 survey and the set of planets already known to orbit stars in our sample. With a larger sample of planets with measured masses in the 3-30 $M_{\oplus}$ range we will measure the mass function for small planets with higher mass resolution. Combining the mass function from this survey with the size distribution from Kepler, we will probe the density and core mass properties of super-Earths to inform formation theories of the galaxy's most abundant planets.

Nearby $G$ and $\mathrm{K}$ dwarf stars are observationally advantageous. High signal-to-noise ratio $(\mathrm{S} / \mathrm{N})$ spectroscopy can often be obtained with relatively short exposure times, facilitating many precise time series RV measurements with sensitivity to planets with small Doppler amplitudes. The stars can be characterized precisely using spectroscopy and asteroseismology (when available) that improve estimates of the star and planet properties. The advantages of nearby, bright targets are critical for characterization of the planets atmospheres transmission, emission, and direct spectroscopy.

Here we present the discovery of three roughly Neptune mass planets orbiting bright stars within $25 \mathrm{pc}$. This paper is structured as follows. Our observational setup and RV measurements are described in Section 2. In Section 3 we discuss our derived stellar properties for each of the three stars and compare with previous literature studies. We describe our methods used to discover these planets in Section 4. We describe our modeling procedure used to obtain the final adopted parameters and their associated uncertainties and our various tests to ensure that the signals are planetary in nature in Section 4.2. We analyze photometry of each of the three systems in Section 5, and conclude with a summary and discussion in Section 6.

\section{RADIAL VELOCITY MEASUREMENTS}

We collected 571 RV measurements of HD 42618 using Keck/HIRES (Vogt et al. 1994) and 35 measurements using the Levy spectrograph on the APF (Radovan et al. 2014; Vogt et al. 2014) over the past 19 years starting in 1996. For each $\mathrm{RV}$ measurement the starlight is passed through a cell of gaseous iodine that imprints a dense forest of molecular absorption lines onto the stellar spectrum and serves as both a wavelength and point-spread function (PSF) reference. We also collected a single set of iodine-free observations of this star that was deconvolved with the instrumental PSF and used as a model of the intrinsic stellar spectrum. Each observation was forward modeled as the intrinsic stellar spectrum Doppler shifted by an arbitrary amount, then multiplied by the transmission of iodine, and convolved with the instrumental PSF modeled as a sum of 13 Gaussians with fixed widths and positions but heights free to vary (Butler et al. 1996). The Levy slit-fed spectrograph also relies on an iodine cell for precise RVs and our observational setup is described in detail in Fulton et al. (2015).

Our setup was identical for the three stars. We collected 328 Keck/HIRES measurements and 73 APF measurements for HD 164922 over the past 19 years. All of the APF measurements and 244 of the Keck measurements are new since the publication of Butler et al. (2006). For HD 143761 we obtained 519 RV measurements using Keck/HIRES and 157 
measurements using the Levy spectrograph on the APF. The Keck observations of HD 143761 started in 2006 and the total observational baseline is 8 years. We do not include the Lick $3.0 \mathrm{~m}$ data from Noyes et al. (1997) and Butler et al. (2006) in our analysis. We find that including the Lick data does not significantly improve the uncertainties on any of the orbital parameters and it adds an additional source of systematic uncertainty that is less well characterized and understood.

Our Doppler pipeline has been tuned in small ways over the years to improve RV precision. Here we describe a new pipeline improvement that decorrelates the measured RVs with nuisance parameters from the model and spectrometer state parameters. This decorrelation offers modest improvements in Doppler precision $\left(\sim 1 \mathrm{~m} \mathrm{~s}^{-1}\right)$ and is only applied to time series $\mathrm{RV}$ of stars for which the number of spectra greatly exceeds the number of potential decorrelation parameters. The nuisance parameters in Doppler analysis include descriptions of the PSF over the spectral format and the wavelength solution. The PSF is parameterized as a sum Gaussians with fixed widths and centers, but variable amplitudes. We also have a wealth of information about the weather and environment inside and outside the spectrograph extracted from the FITS headers of the raw APF spectra. Some environmental information is available in the FITS headers for the Keck data, but we have not yet implemented a system to extract these values for the tens of thousands of Keck spectra taken over the last 20 years. For Keck data we only include the nuisance parameters that are part of the forward modeling process. We clean the RVs of systematic trends by removing any correlations that these parameters show with the final RVs. We search for significant correlations of the RVs with all of the PSF parameters by calculating the Spearman rank correlation coefficient (Spearman 1904) and flag any parameters that show correlation coefficients greater than 0.1. The flagged parameters are included in a multivariate ordinary least squares linear regression using the STATSMODELS ${ }^{21}$ package in Python. This model for RV as a function of all parameters included in the fit is then subtracted from the raw RVs. This process is done blindly in the planet discovery/identification phase but once planet candidates are identified in a given dataset we first model the system to find the best fit N-planet Keplerian model then perform the detrending procedure on the residuals to this model. The detection of all newly discovered planets in this work does not depend on this detrending and they are easily identified at high significance in either the detrended or nondetrended datasets.

We reject measurements with low signal-to-noise ratios (S/ $\mathrm{N}<60$ per pixel) and/or uncertainties more then $9 \sigma$ larger then the median uncertainty, which results in the omission of $<1 \%$ of the data for each of the three stars. Since these stars are exceptionally bright we almost always collect three consecutive measurements in order to average out RV shifts caused by $p$ mode oscillations (Dumusque et al. 2011). The three measurements are then binned together before the stellar jitter is added in quadrature during the modeling process (see Section 4.2). This effectively reduces the weight of the three measurements to that of a single measurement, but averages out some of the astrophysical noise in the process and prevents time-correlated instrumental systematic noise from biasing the results. We also extract the $\mathrm{Ca}$ II $\mathrm{H}$ and $\mathrm{K}$ activity index $\left(S_{\mathrm{HK}}\right)$ using the

\footnotetext{
${ }^{21}$ https://pypi.python.org/pypi/statsmodels
}

Table 1

Radial Velocities

\begin{tabular}{lccccc}
\hline \hline $\mathrm{HD}$ & $\begin{array}{c}\mathrm{BJD}_{\mathrm{TDB}} \\
(-2440000)\end{array}$ & $\begin{array}{c}\mathrm{RV}^{\mathrm{a}} \\
\left(\mathrm{m} \mathrm{s}^{-1}\right)\end{array}$ & $\begin{array}{c}\text { Unc. } \\
\left(\mathrm{m} \mathrm{s}^{-1}\right)\end{array}$ & Inst. $^{\mathrm{b}}$ & $S_{\mathrm{HK}}{ }^{\mathrm{c}}$ \\
\hline 42618 & 2450366.126333 & +2.85 & 1.12 & $k$ & $\cdots$ \\
42618 & 2453694.093412 & +1.17 & 1.13 & $j$ & 0.161 \\
164922 & 2454777.744397 & -9.19 & 1.04 & $j$ & 0.152 \\
164922 & 2457267.662041 & -4.43 & 1.90 & $a$ & 0.145 \\
143761 & 2455455.762546 & -41.98 & 1.27 & $j$ & 0.149 \\
143761 & 2457292.685768 & -8.08 & 2.92 & $a$ & 0.135 \\
\hline
\end{tabular}

Notes.

${ }^{a}$ Zero point offsets between instruments have not been removed and must be fit as free parameters when analyzing this dataset.

${ }^{\mathrm{b}} k=$ pre-upgrade Keck/HIRES, $j=$ post-upgrade Keck/HIRES, $a=$ APF.

${ }^{\mathrm{c}}$ Uncertainties on $S_{\mathrm{HK}}$ are 0.002 for all Keck measurements and 0.004 for all APF measurements.

(This table is available in its entirety in machine-readable form.)

technique of Isaacson \& Fischer (2010) for every RV measurement on both Keck and APF, however there may be an arbitrary zero point offset in the $S_{\mathrm{HK}}$ values between the Keck and APF values. The uncertainties for the $S_{\mathrm{HK}}$ measurements are systematically limited to 0.002 for Keck and 0.004 for APF. This was estimated by measuring the standard deviation of all measurements of the extremely chromospherically quiet star, HD 10700. All RV measurements and the associated $S_{\mathrm{HK}}$ values can be found in Table 1 . We include only the detrended velocities in Table 1 but the full set of environmental and PSF parameters for each observation along with the non-detrended velocities can be downloaded from https://github.com/bjfultn/three_neptunes.

\section{STELLAR PROPERTIES}

\section{1. $H D 42618$}

HD 42618, also known as HIP 29432 and Gl 3387, is a well studied solar analogue located at a distance of $23.5 \mathrm{pc}$ (van Leeuwen 2007). The star was not previously known to host any exoplanets. We analyzed 5 high S/N Keck-HIRES spectra (described below) using SpecMatch (Petigura 2015) to obtain the mean spectroscopic parameters listed in Table 2. SpecMatch uses trilinear interpolation to synthesize high resolution model spectra from the Coelho (2014) grid of models for any set of arbitrary stellar parameters $\left(T_{\text {eff }}, \log g\right.$, $[\mathrm{Fe} / \mathrm{H}]$, and $v \sin i$ ) that are contained within the limits of the model grid. The interpolated models are then compared to the observed spectrum. We maximize the likelihood $\left(\mathcal{L}=e^{-\chi^{2} / 2}\right)$ to determine the optimal stellar parameters, where $\chi^{2}$ is summed over the extracted spectral pixels and normalized by the flux uncertainties.

Our spectral analysis is consistent with the results of Valenti \& Fischer (2005) who extracted spectroscopic parameters using Spectroscopy Made Easy (SME) and found $T_{\text {eff }}=5747 \pm 44 \mathrm{~K}$, $\log g=4.43 \pm 0.06$, and $[\mathrm{Fe} / \mathrm{H}]=-0.11 \pm 0.03$. HD 42618 is chromospherically quiet with $\log R_{\mathrm{HK}}^{\prime}=-5.01$ (Isaacson \& Fischer 2010). It was deemed to be a good solar-analog based on a very similar chemical abundance pattern to the Sun (Morel et al. 2013). Those authors also derive $T_{\text {eff }}=5765 \pm 17 \mathrm{~K}$, and $\log g=4.48 \pm 0.04$, consistent with our SpecMatch results. Ramírez et al. (2014) measured the fundamental parameters of HD 42618 differentially relative to the Sun which allowed them to 
Table 2

Adopted Stellar Properties

\begin{tabular}{lccc}
\hline \hline Parameter & HD 42618 & HD 164922 & HD 143761 \\
\hline $\begin{array}{l}\text { Spectral } \\
\text { type }\end{array}$ & $\mathrm{G} 4 \mathrm{~V}^{1}$ & $\mathrm{G} 9 \mathrm{~V}^{6}$ & ${\mathrm{G} 0 \mathrm{~V}^{8}}^{8}$ \\
$B-V(\mathrm{mag})$ & $0.657^{2}$ & $0.800^{7}$ & 0.600 \\
$V(\mathrm{mag})$ & $6.839^{2}$ & $6.99^{7}$ & $5.41^{8}$ \\
$J$ (mag) & $5.701 \pm 0.023^{3}$ & $5.553 \pm 0.026^{3}$ & $4.09^{3}$ \\
$H(\mathrm{mag})$ & $5.385 \pm 0.024^{3}$ & $5.203 \pm 0.017^{3}$ & $3.99^{3}$ \\
$K(\mathrm{mag})$ & $5.301 \pm 0.020^{3}$ & $5.113 \pm 0.020^{3}$ & $3.89 \pm 0.05^{8}$ \\
Distance $(\mathrm{pc})$ & $23.50 \pm 0.30^{4}$ & $22.13 \pm 0.27^{4}$ & $17.236 \pm 0.024^{4}$ \\
$T_{\mathrm{eff}}(\mathrm{K})$ & $5727 \pm 60$ & $5293 \pm 32$ & $5627 \pm 54^{11}$ \\
$\log g(\mathrm{cgs})$ & $4.44 \pm 0.07$ & $4.387 \pm 0.014$ & $4.121 \pm 0.018$ \\
{$[\mathrm{Fe} / \mathrm{H}](\mathrm{dex})$} & $-0.09 \pm 0.04$ & $+0.16 \pm 0.05$ & $-0.31 \pm 0.05$ \\
$v$ sin $i\left(\mathrm{~km} \mathrm{~s}{ }^{-1}\right)$ & $\leqslant 2$ & $\leqslant 2$ & $\leqslant 2$ \\
$L_{\star}\left(L_{\odot}\right)$ & $0.98 \pm 0.17$ & $0.703 \pm 0.017$ & $1.706 \pm 0.042^{11}$ \\
$M_{\star}\left(M_{\odot}\right)$ & $1.015 \pm 0.061^{12}$ & $0.874 \pm 0.012$ & $0.889 \pm 0.030$ \\
$R_{\star}\left(R_{\odot}\right)$ & $0.999 \pm 0.087^{12}$ & $0.999 \pm 0.017$ & $1.3617 \pm 0.0262^{11}$ \\
$\log R_{\mathrm{HK}}^{\prime}$ & $-5.01^{5}$ & $-5.06^{5}$ & $-5.05^{5}$ \\
$S_{\mathrm{HK}}$ & $0.157^{5}$ & $0.154^{5}$ & $0.150^{5}$ \\
\hline
\end{tabular}

References. (1) Medhi et al. (2007), (2) Koen et al. (2010), (3) Cutri et al. (2003), (4) van Leeuwen (2007), (5) Isaacson \& Fischer (2010), (6) Gray et al. (2003), (7) Butler et al. (2006), (8) van Belle \& von Braun (2009), (9) Noyes et al. (1997), (10) van Belle \& von Braun (2009), (11) von Braun et al. (2014), (12) Torres et al. (2010).

obtain highly precise values for $T_{\text {eff }}=5758 \pm 5 \mathrm{~K}$, $\log g=4.44 \pm 0.01$, and $[\mathrm{Fe} / \mathrm{H}]=-0.096 \pm 0.005$ that show good agreement with our results. It has also been noted that HD 42618 shows a low lithium abundance of $A_{\mathrm{Li}}=1.22$ (Ramírez et al. 2012) similar to that of the Sun $\left(A_{\mathrm{Li}}=1.05 \pm 0.1\right.$ Asplund et al. 2009). Our adopted stellar mass and radius for HD 42618 are based on the relations of Torres et al. (2010) using our spectroscopic constraints on $T_{\text {eff }}, \log g$, and $[\mathrm{Fe} / \mathrm{H}]$. HD 42618 was also target of the CoRoT mission (Baglin et al. 2009), with a preliminary detection of solar-like oscillations presented by Barban et al. (2013). We performed an independent asteroseismic analysis of the CoRoT photometry (see Section 5.1.1), which yielded a mass and radius in agreement with our adopted values.

\section{2. $H D 164922$}

HD 164922, also known as HIP 88348 and Gl 9613, is a bright, chromospherically inactive $\left(\log R_{\mathrm{HK}}^{\prime}=-5.06\right.$, Isaacson \& Fischer 2010) G9 V dwarf located 22.1 pc away (van Leeuwen 2007). It was previously known to host a single Saturn-mass planet orbiting with a semimajor axis of 2.1 au (Butler et al. 2006). This target was one of several selected for more intensive long-term RV monitoring by Keck/HIRES based on both the stellar properties, and the mass and orbit of the previously detected planet making the system particularly well-suited for detecting additional low-mass planets. It was also on the Eta-Earth target list as part of a deep Doppler survey for low-mass planets (Howard et al. 2010).

We measured the stellar radius for HD 164922 using the CHARA Array. Interferometric observations of HD 164922 were taken on 2012 May 13 and 14 using the Pavo beam combiner (ten Brummelaar et al. 2005; Ireland et al. 2008). Observations of the science target were interleaved with the calibrator stars HD 164900, HD 161019, and HD 165373 (Bonneau et al. 2006, 2011). The data were reduced and

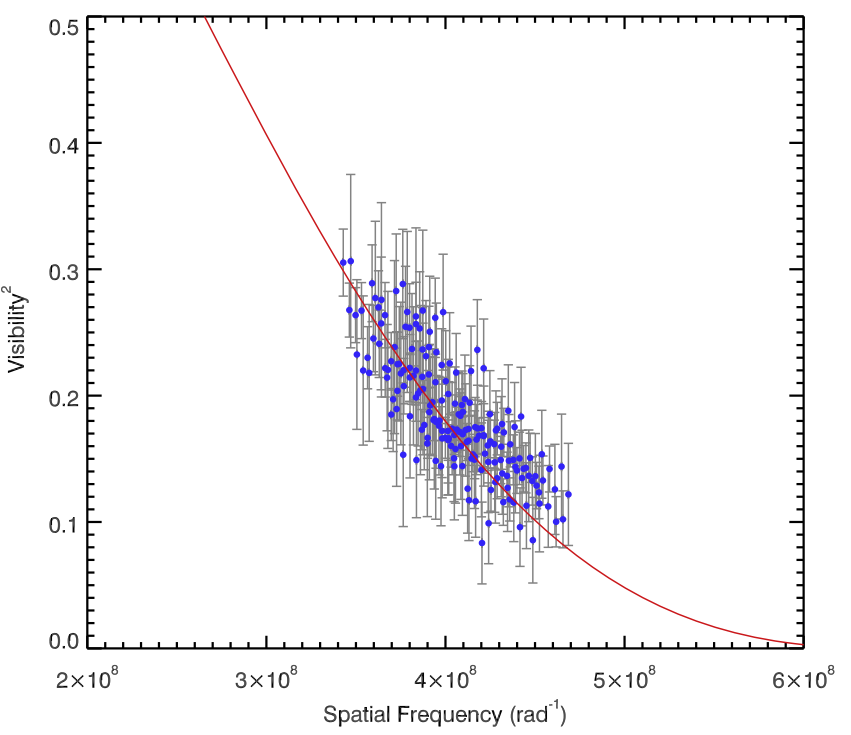

Figure 1. Observed squared visibility vs. spatial frequency for HD 164922 (blue diamonds). The red line shows the best fit limb-darkened model. See Section 3.2 for a discussion of the implications of this figure.

calibrated using the standard data reduction pipeline (for details see White et al. 2013). We use the $R$-band limb-darkening coefficient from Claret \& Bloemen (2011), $\mu_{R}=0.633$, to determine a limb-darkened angular diameter $\theta_{\mathrm{UD}}=$ mas (Figure 1).

Combining the angular diameter with the parallax yields a stellar radius of $0.999 \pm 0.017 R_{\odot}$. We determine a stellar bolometric flux of $F_{\mathrm{Bol}}=4.61 \pm 0.03 \mathrm{erg} \mathrm{s}^{-1} \mathrm{~cm}^{-2}$ by fitting a spectral template from Pickles (1998) to flux calibrated photometry after applying revised filter profiles from Mann \& von Braun (2015). This translates to a luminosity $L=0.703 \pm 0.017 L_{\odot}$. Lastly, we use our measured angular diameter with the star's bolometric flux to derive an empirical effective temperature $T_{\text {eff }}=5293 \pm 32 \mathrm{~K}$.

We then use the interferometrically determined parameters to inform a SpecMatch analysis of a stack of 5 high $\mathrm{S} / \mathrm{N}$ APF spectra using an iterative technique. An initial uninformed SpecMatch analysis of the APF spectra (all priors uniform) gives $T_{\text {eff }}=5318 \pm 70 \mathrm{~K}, \log g=4.36 \pm 0.08$ and $[\mathrm{Fe} / \mathrm{H}]$ $=0.17 \pm 0.05$. We use this spectroscopically measured [Fe/H] combined with the $T_{\text {eff }}$ and $R_{\star}$ determined from the CHARA data along with existing $J, H$, and $K$ photometry (Cutri et al. 2003) as Gaussian priors in a fit to Dartmouth isochrones (Dotter et al. 2008) using the isochrones package (Morton 2015). ${ }^{22}$ This gives the first estimate of the full set of stellar parameters. We then re-run SpecMatch with Gaussian priors applied to $T_{\text {eff }}$ and $\log g$ from the isochrones output. The full likelihood with the Gaussian priors is

$$
\begin{aligned}
\mathcal{L}= & \exp \left[-\frac{1}{2}\left(\chi^{2}+\left(\frac{T_{\text {eff }}-5293 \mathrm{~K}}{32 \mathrm{~K}}\right)^{2}\right.\right. \\
& \left.\left.+\left(\frac{\log g-4.387}{0.014}\right)^{2}\right)\right] .
\end{aligned}
$$

\footnotetext{
22 https://github.com/timothydmorton/isochrones
} 
In this case the changes to stellar parameters become negligible after two iterations and the process is halted. The resulting stellar parameters are listed in Table 2 .

Since the star is bright and already a known planet host it has been the subject of many spectroscopic studies. Santos et al. (2013) find $T_{\text {eff }}=5356 \pm 45 \mathrm{~K}, \log g=4.34 \pm 0.08$, and $[\mathrm{Fe} / \mathrm{H}]=+0.14 \pm 0.03$, all consistent with our analysis to within $1 \sigma$. Valenti \& Fischer (2005) find a significantly higher value for $\log g=4.51 \pm 0.06$ and $T_{\text {eff }}=5385 \pm 44 \mathrm{~K}$ but a consistent metallicity value of $[\mathrm{Fe} / \mathrm{H}]=+0.17 \pm 0.03$. Ghezzi et al. (2010) measure $T_{\text {eff }}=5378 \pm 50 \mathrm{~K}$, $\log g=4.30 \pm 0.22$, and $[\mathrm{Fe} / \mathrm{H}]=+0.21 \pm 0.03$ also using high-resolution spectroscopy which, except for $T_{\text {eff }}$, is also consistent with our analysis to within $1 \sigma$. As seen by the range of $\log g$ values obtained by these various studies, it can be difficult to pin down the stellar gravity from high resolution spectra alone. Since our $\log g$ value is constrained via the direct measurement of the stellar radii from interferometry we adopt the new slightly lower value for $\log g$.

\subsection{HD 143761}

HD 143761 is the closest and brightest star of the three studied in this work. The star is also known as $\rho$ Corona Borealis, HIP 78459, and Gl 9537. It is a slightly evolved naked eye $(V=5.41)$ G0 V star (van Belle \& von Braun 2009) located at a distance of 17.236 pc (van Leeuwen 2007). It was previously known to host a warm Jupiter-mass planet with an orbital period of 39 days (Noyes et al. 1997). This star was also part of the Eta-Earth survey and was independently selected for intensive long-term RV monitoring based on both the stellar properties, and the mass and orbit of the previously detected planet. Like HD 42618 and HD 164922 this star is chromosherically quiet with $R_{\mathrm{HK}}^{\prime}=-5.05$ (Isaacson \& Fischer 2010). As with HD 164922 we performed an iterative interferometric+spectroscopic analysis using the CHARA results from von Braun et al. (2014) and a stack of 5 high S/ $\mathrm{N}$ APF spectra. The likelihood was essentially the same as Equation (1) but with the $T_{\text {eff }}$ and $\log g$ values for HD 143761 substituted in the last two terms.

Valenti \& Fischer (2005) measure $T_{\text {eff }}=5822 \pm 44 \mathrm{~K}$, and Fuhrmann et al. (1998) measure $T_{\text {eff }}=5821 \pm 20 \mathrm{~K}$ both using high resolution spectroscopy. Both of these values are significantly hotter then our adopted value of $T_{\text {eff }}=5627 \pm 54 \mathrm{~K}$ from von Braun et al. (2014). We chose to adopt the value from von Braun et al. (2014) in order to maintain self-consistency with the interferometrically measured stellar radius and luminosity. Our metallicity value is also consistent within $1 \sigma$ to that of Fuhrmann et al. (1998) and but is significantly lower than that of Valenti \& Fischer (2005). Valenti \& Fischer (2005) again measure a significantly higher value for $\log g=4.36 \pm 0.06$, but our $\log g=4.121 \pm 0.018$ value is consistent with $\log g=4.12 \pm 0.1$ from Fuhrmann et al. (1998).

\section{KEPLERIAN ANALYSIS}

\subsection{Discovery}

We discovered each of the three new planets and rediscovered the previously known planets using a technique essentially identical to that of Fulton et al. (2015). In brief, we calculate a two-dimensional Lomb-Scargle periodogram
(2DKLS, O'Toole et al. 2009) to look for significant periodic signals that are well fit by a Keplerian orbital model. Our implementation of the 2DKLS periodogram incorporates arbitrary zero-point offsets between each instrument. The periodogram power $(Z)$ represents the improvement to the $\chi^{2}$ statistic relative to that of a baseline fit. When searching for the first planet in a system the baseline fit is simply a flat line or linear trend. If any significant signals are found after the first iteration the baseline model then becomes the single planet Keplerian model and we calculate the improvement to $\chi^{2}$ when a second planet is added, without subtracting the first. We repeat this process until no more significant peaks are found in the 2DKLS periodogram. We start the search assuming no known signals in order to ensure that the previously published planets can be automatically detected using our pipeline. An initial jitter term of $2.0 \mathrm{~m} \mathrm{~s}^{-1}$ is added in quadrature with the RVs before starting the 2DKLS search in order to ensure fair weighting between the Keck and APF data sets. We expect both data sets to be limited by instrumental/astrophysical systematics rather than photon noise.

The discovery pipeline is completely automated in order to facilitate injection recovery tests that will allow us to characterize the pipeline completeness for future occurrence analysis (Howard \& Fulton 2016). We calculate an empirical periodogram false alarm probability (eFAP) by fitting a power law to the distribution of periodogram values between the 50th and 97th quartiles. This fit provides an estimate of the significance of periodogram peaks of a given value. When multiplied by the number of independent test periods, the fit gives the approximate probability that we would find a peak of a given value within any particular periodogram. Any periodogram peak with an eFAP below $0.1 \%$ is automatically considered a viable candidate and the search is continued until no more periodogram peaks fall above the $0.1 \%$ eFAP threshold. Further details of the automated planet detection pipeline can be found in Howard \& Fulton (2016). We note that the eFAP metric is used simply to automatically identify candidates. The significance of the corresponding periodogram peaks are checked using the bootstrapping technique described in Section 4.3. Each of the previously known planets were rediscovered with eFAPs much less than those of the new planets announced in this work.

We discover two significant signals in the RV time series of HD 42618. One with a long period of 4850 days, and a second at a period of 149.6 days. Upon inspection of the $\mathrm{Ca}$ II $\mathrm{H}$ and $\mathrm{K}$ activity index time series we notice that this index shows a periodicity with a period very similar to that of the long period RV signal. The period of 4850 days is also very similar to the period of the Sun's magnetic activity cycle. We conclude that this is likely the signature of the stellar magnetic activity cycle and not the signature of an orbiting planet. We include this long-period signal as an additional eccentric Keplerian in all further modeling. HD $42618 \mathrm{~b}$ is easily detected in the Keck data alone and the combined Keck + APF dataset but we do not yet have enough APF measurements to detect it in the APF data alone. Figure 2 shows the most likely model from the posterior of the two-Keplerian model, the 2DKLS periodogram used to discover HD $42618 \mathrm{~b}$, and the RVs phased to the orbital period of planet $b$.

Wright et al. (2007) mentioned a candidate planetary signal with a period of 75.8 days and $K=3 \mathrm{~m} \mathrm{~s}^{-1}$ orbiting HD 

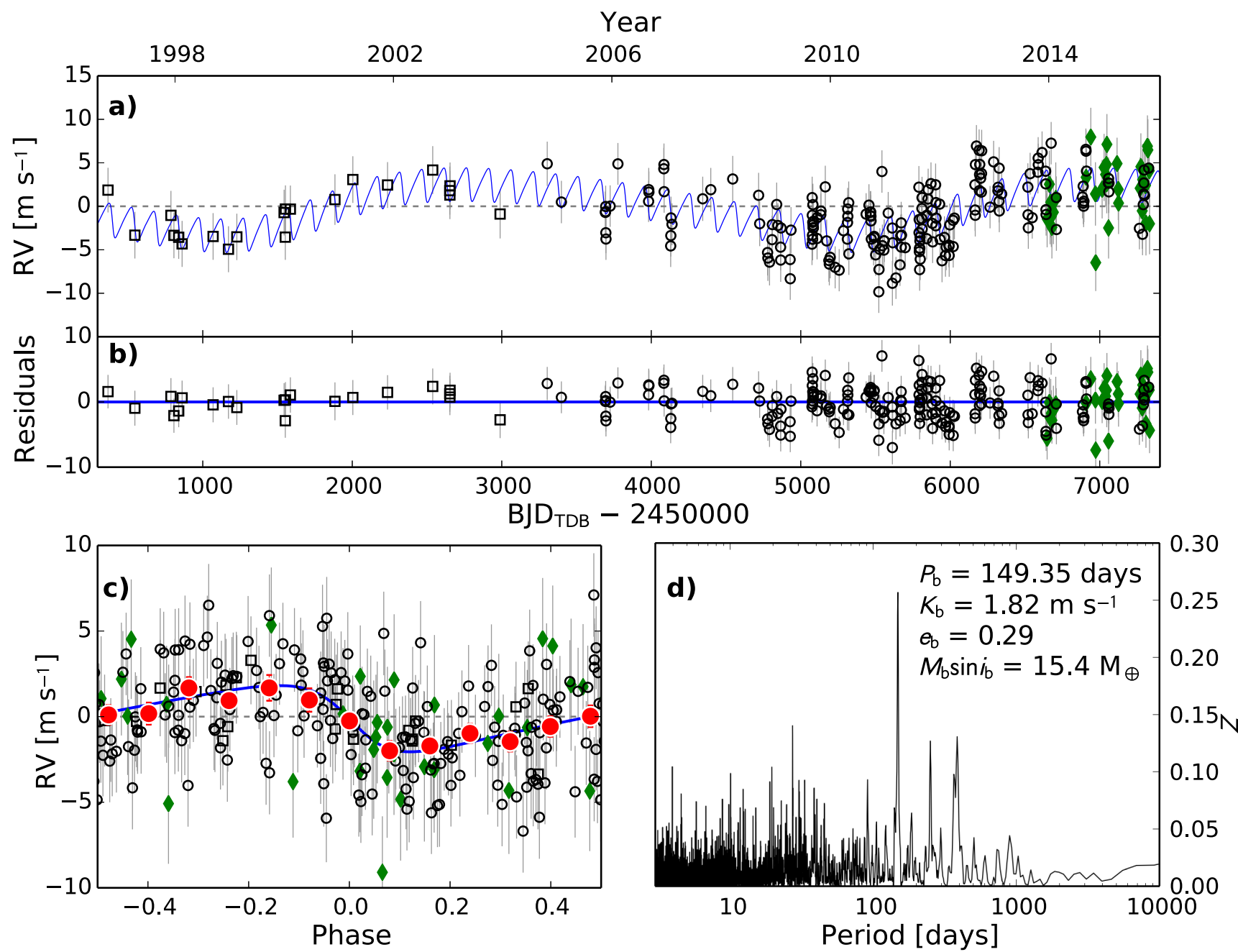

Figure 2. One-planet Keplerian orbital model plus one additional long-period Keplerian to model the stellar magnetic activity cycle for HD 42618 . The most likely model is plotted but the orbital parameters annotated on the figure and listed in Tables 3 and 6 are the median values of the posterior distributions. The process used to find the orbital solution is described in Section 4.2. (a) Full binned RV time series. Open black squares indicate pre-upgrade Keck/HIRES data (see Section 2), open black circles are post-upgrade Keck/HIRES data, and filled green diamonds are APF measurements. The thin blue line is the most probable 1-planet plus stellar activity model. We add in quadrature the RV jitter term listed in Table 3 with the measurement uncertainties for all RVs. (b) Residuals to the most probable 1-planet plus stellar activity model. (c) Binned RVs phase-folded to the ephemeris of planet b. The long-period stellar activity signal has been subtracted. The small point colors and symbols are the same as in panel (a). For visual clarity, we also bin the velocities in 0.08 units of orbital phase (red circles). The phase-folded model for planet $b$ is shown as the blue line. (d) 2DKLS periodogram showing the improvement to $\chi^{2}$ for a model including the long period activity signal and a single planet compared to a model that only includes the activity signal.

164922 but did not have sufficient data to claim a significant detection. With seven years of additional Keck data, and 2 years of APF data we can firmly establish this signal as being coherent and persistent as expected for the Doppler motion caused by an orbiting planet. The short period planet is easily detected in either the APF or Keck data alone. The long-period planet can only be detected in the long baseline Keck data but we do observe a linear RV trend that emerged during the most recent APF observing season which is a result of the massive outer planet. Figures 3 and 4 show the most likely model from the posteriors for the two planet Keplerian model and the 2DKLS periodograms used to discover/re-discover each of the two planets.

We discover a super-Neptune mass planet orbiting HD 143761 exterior to the known Jupiter mass planet that has an orbital period of 39 days. The new planet has an orbital period of 102 days and a semi-amplitude of $3.7 \mathrm{~m} \mathrm{~s}^{-1}$. Each planet is discovered with very high significance in both the Keck and Keck +APF datasets individually. The most likely model from the posteriors for the two planet model and the 2DKLS detection periodograms are shown in Figure 5.

\subsection{Characterization}

We estimated orbital parameters and their associated uncertainties using the ExoPy Differential Evolution Markov Chain Monte Carlo (DE-MCMC, Ter Braak 2006) modeling code and a technique identical to that of Fulton et al. (2013), Knutson et al. (2014), and Fulton et al. (2015).

Our multi-planet RV model is a sum of Keplerian singleplanet models over all planets in the system. For each singleplanet Keplerian model (indexed by $i$ ) we compute posteriors for the orbital period $\left(P_{i}\right)$, time of inferior conjunction $\left(T_{\mathrm{conj}, i}\right)$, eccentricity $\left(e_{i}\right)$, argument of periastron of the star's orbit $\left(\omega_{i}\right)$, 

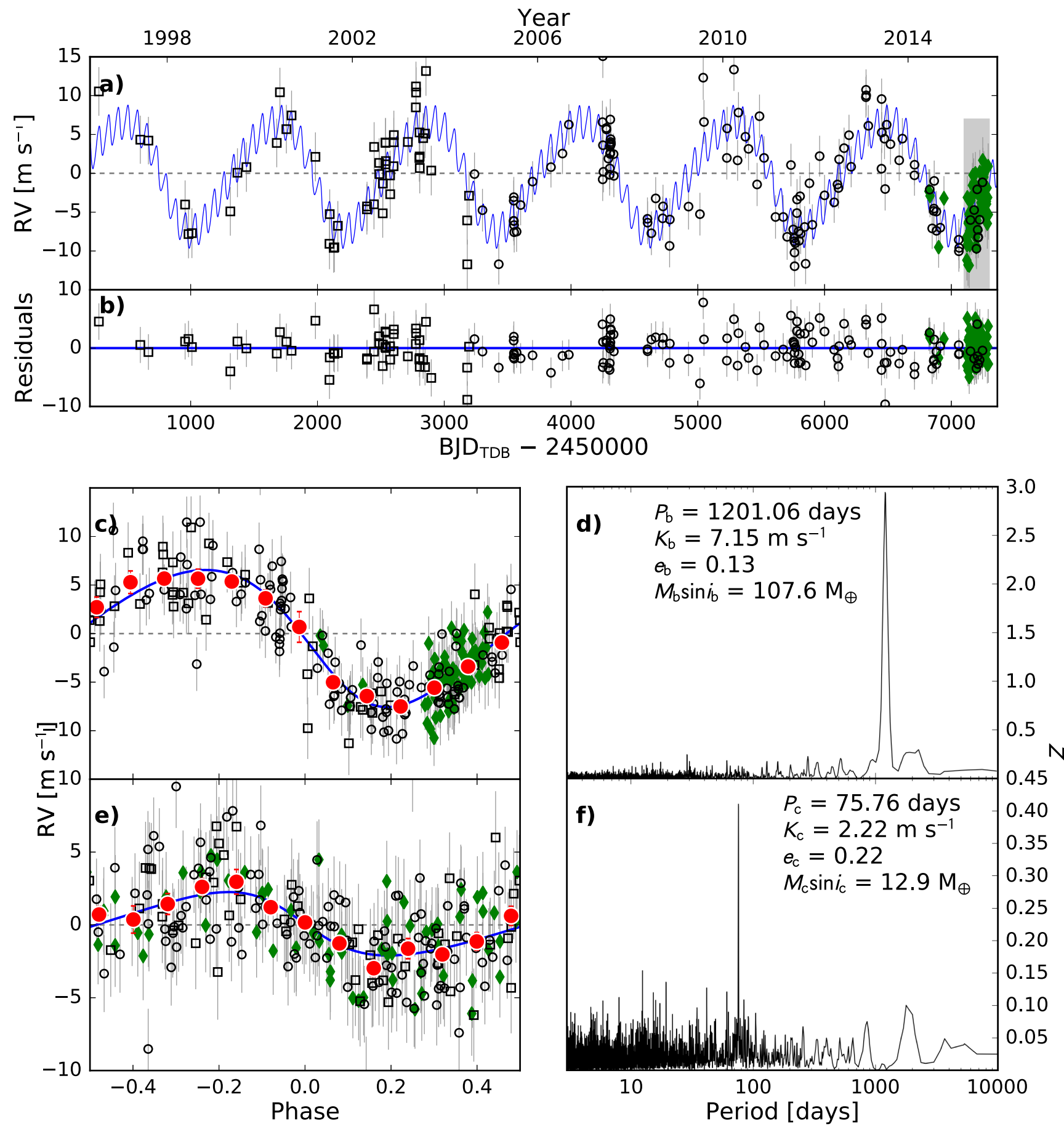

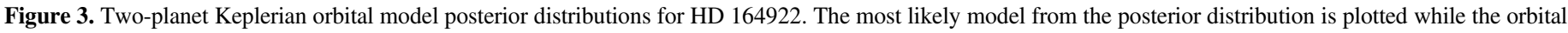

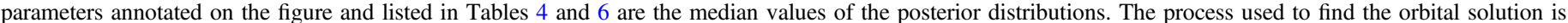

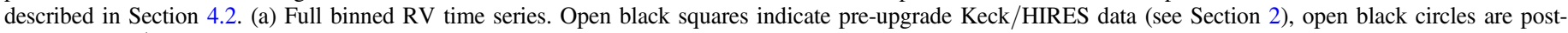

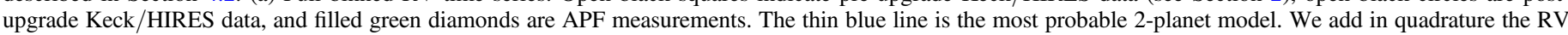

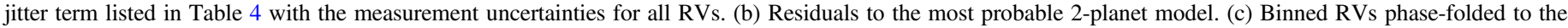

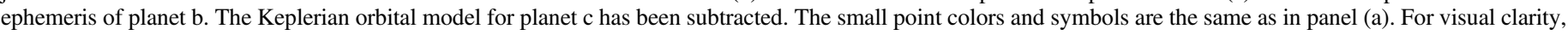

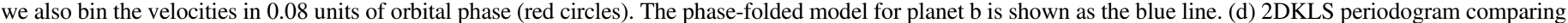

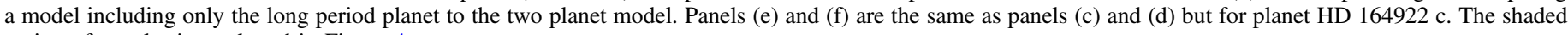
region of panel a is re-plotted in Figure 4.

velocity semi-amplitude $\left(K_{i}\right)$. We also compute posteriors for the offsets between pre-upgrade, post-upgrade, and the APF datasets $(\gamma)$, and an RV jitter term with the specific prior described in Fulton et al. (2015) and Johnson et al. (2011). In order to speed convergence, we choose to re-parameterize some of the physical parameters as $\sqrt{e} \cos \omega, \sqrt{e} \sin \omega$, and $\log K$. 


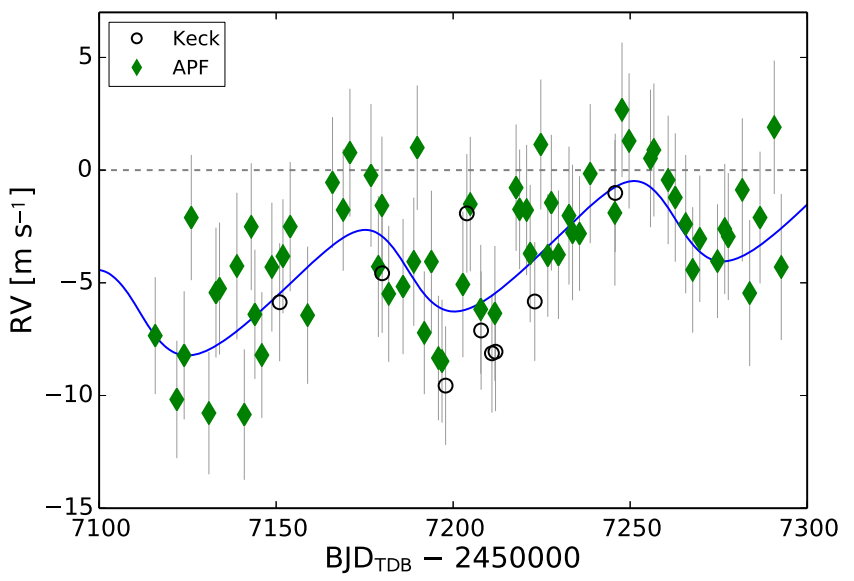

Figure 4. Recent RVs for HD 164922 highlighted in the gray box of Figure 3 panel (a) featuring the high cadence APF observations collected during the most recent observational season.

The full likelihood for this Keplerian model is

$$
\begin{aligned}
\mathcal{L}= & \exp \left(-\frac{1}{2} \sum_{n}\left[\frac{\left(v_{n}-M_{n}\right)^{2}}{\sigma_{\mathrm{v}, n}^{2}+\sigma_{\mathrm{jitt}}^{2}}\right.\right. \\
& \left.\left.+2 \ln \left(\sqrt{2 \pi}\left(\sigma_{\mathrm{v}, n}^{2}+\sigma_{\mathrm{jitt}}^{2}\right)^{1 / 4}\right)\right]\right)
\end{aligned}
$$

summed over $n$ RV measurements $\left(\mathrm{v}_{n}\right)$ with associated uncertainties $\sigma_{\mathrm{v}, n} . M_{n}$ is the Keplerian model for observation $n$. We assign uniform priors to $\log P, T_{\text {conj }}, \sqrt{e} \cos \omega, \sqrt{e} \sin \omega$, $\log K$, and $\gamma$ for each instrument. We follow the prescription of Eastman et al. (2013) checking for convergence at regular intervals during the MCMC runs by calculating the GelminRubin statistic and the number of independent draws $\left(T_{Z}\right.$, Ford 2006). We consider the chains well mixed and halt the MCMC run when the Gelmin-Rubin statistic is within $1 \%$ of unity and $T_{Z}>1000$ for all free parameters. All of the adopted median values and $68 \%$ confidence intervals of the posterior distributions are listed in Tables 3-5.

\subsection{Bootstrap False Alarm Assessment}

We conduct a bootstrap false alarm assessment to verify and double check that the periodogram peaks with low eFAPs are indeed statistically significant periodic signals and not caused by random fluctuations of noise. For all three stars we scramble the RV time series 1000 times and recalculate the 2DKLS periodogram searching for $\mathrm{N}+1$ planets where $\mathrm{N}$ is the number of previously published planets in the system. We record the highest periodogram value from each trial and plot the distribution of periodogram peak heights relative to the periodogram peak values corresponding to the newly discovered planets. These distributions are plotted in Figure 6. The periodogram peak heights corresponding to each of the new planets are well separated from the distribution of peaks in the scrambled RV trials. This indicates that the probability that random noise could conspire to create the periodogram peaks used to detect the planets are $<0.1 \%$. However, a visual inspection of the distribution of periodogram peak heights in Figure 6 suggests that the FAPs are likely much lower.

\subsection{Brown Dwarf Companion to HD 143761?}

HD $143761 \mathrm{~b}$ was one of the first exoplanets discovered (Noyes et al. 1997). Gatewood et al. (2001) later reported that they detected the signature of HD $143761 \mathrm{~b}$ in astrometric data from the Multichannel Astrometric Photometer and Hipparcos. Zucker \& Mazeh (2001) were quick to point out that the statistical significance of this astrometric detection is only $2 \sigma$. Over a decade after the discovery of HD 143761 b, Reffert \& Quirrenbach (2011) claimed to detect the same astrometric signal of the warm Jupiter in a re-reduction of Hipparcos data. The astrometric orbit suggests that the system is nearly face on with an inclination between 0.4 and 0.7 . This would imply that, after correcting for the viewing angle, HD $143761 \mathrm{~b}$ is not a planet but instead a low mass $M$ star with $100<M_{b}<200 M_{J}$.

\subsubsection{Interferometry of $H D 143761$}

Long-baseline interferometry is sensitive to some stellar binaries. HD 143761 was observed interferometrically with the CHARA Array (von Braun et al. 2014). If we assume a face-on orbit of HD $143761 \mathrm{~b}$ at a distance of $17.2 \mathrm{pc}$, then the angular distance between it and the principal component is 14 milliarcseconds independent of phase angle. This is detectable as a separated fringe packet at $H$-band with CHARA (Farrington et al. 2010), provided the brightness contrast is not larger than $\Delta H \sim 2$ (Farrington et al. 2010; Raghavan et al. 2012).

Using the mass constraints from the astrometric orbit, a comparison with the Dartmouth isochrones for metallicities spanning the $1 \sigma[\mathrm{Fe} / \mathrm{H}]$ uncertainty of $\mathrm{HD} 143761$ from Table 2 corresponds to an apparent $H$ magnitude at a distance of $17.2 \mathrm{pc}$ of around 10. This implies a $\Delta H \simeq 6$, which is significantly below what could be detected as a separate component in CHARA data (cf. equation A5 in Boyajian et al. 2008). Since component c's apparent $H$ magnitude is much fainter than component b's, its detection is impossible in the CHARA data. As expected, an inspection of the CHARA data used in von Braun et al. (2014) did not yield any indication of additional fringe packets.

We also obtained imaging observations of $\rho \mathrm{CrB}$ using the Differential Speckle Survey Instrument (DSSI) on GeminiNorth during the nights of 2014 July 19, 24, and 25. The DSSI camera is a dual-channel speckle imaging system, expounded upon in more detail by Horch et al. (2009), Horch et al. (2011). Observations were acquired using red and near-infrared filters centered on $692 \mathrm{~nm}$ and $880 \mathrm{~nm}$ respectively. Our instrument setup is the same as that described in Horch et al. (2012) and our analysis methodology is outlined by Kane et al. (2014). Briefly, we estimate the limiting magnitude $\Delta m$ (difference between local image maxima and minima) as a function of target separation resulting in a $5 \sigma$ detection curve. More details on the derivation of the DSSI detection limits can be found in Howell et al. (2011). All of our DSSI $\rho$ CrB observations show no evidence of a stellar companion to the host star. Figure 7 shows the detection curve from the $880 \mathrm{~nm}$ image acquired for $\rho \mathrm{CrB}$ on the night of 2014 July 25. The dashed curve is the cubic spline interpolation of the $5 \sigma$ detection limit from 0 ." 1 to 1 !' 2 . The results exclude companions with $\Delta m \sim 5.2$ and $\Delta m \sim 7.5$ at separations of 0 !! 1 and 1.4 respectively. Given the distance of $\rho \mathrm{CrB}$ of $17.236 \mathrm{pc}$, these angular separations correspond to a physical exclusion range of $1.7-24.1 \mathrm{au}$. We can thus rule out stellar companions in close proximity to the 

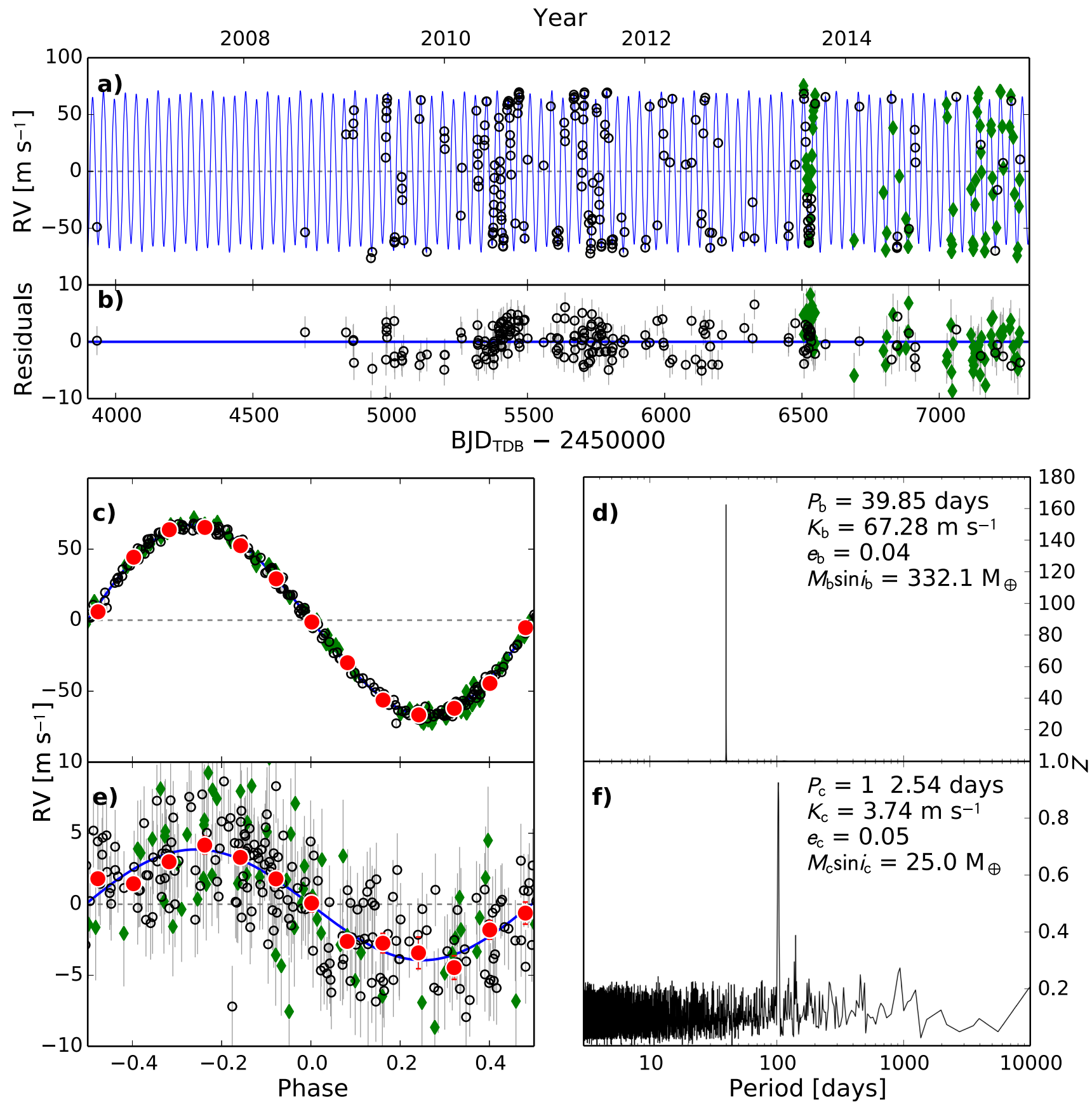

Figure 5. Same as Figure 3 but for planets HD 143761 b and c.

host star, supporting the evidence that the system is not a faceon triple star system, but instead a multi-planet system viewed at moderate to high inclination.

\subsubsection{Stability of the HD 143761 System}

Two planet systems on circular orbits are likely to be unstable if $\Delta<2 \sqrt{3}$ Gladman (1993) for all mutual inclinations, where $\Delta=\frac{a_{\mathrm{in}}-a_{\text {out }}}{R_{\mathrm{H}}}$ and $R_{\mathrm{H}}$ is the Hill radius,

$$
R_{\mathrm{H}}=\left(\frac{M_{\mathrm{in}}+M_{\mathrm{out}}}{3 M_{\star}}\right)^{1 / 3} \frac{a_{\text {in }}+a_{\text {out }}}{2} .
$$

Sky-projected inclinations smaller than $4^{\circ}$ combined with the $M \sin i$ constraints listed in Table 6 imply large companion masses and push $\Delta$ below the $2 \sqrt{3}$ stability threshold. If we assume that the system is dynamically stable then the presence of HD 143761 c rules out the low inclination orbit found by Gatewood et al. (2001), and Reffert \& Quirrenbach (2011).

HD 164922 b and c are widely separated and intuitively we would expect them to be in a stable configuration. For completeness, we calculate $\Delta=26.3$ for the minimum masses which suggests that this system configuration is likely to be dynamically stable for a long time.

\subsection{Additional Planet Candidates}

There is an additional significant periodic signal in the RV data for HD 42618 at a period of 388 days and a velocity semi- 
Table 3

Orbital Parameters for HD 42618

\begin{tabular}{|c|c|c|}
\hline Parameter & Value & Units \\
\hline \multicolumn{3}{|c|}{ Modified DE-MCMC Step Parameters ${ }^{\mathrm{a}}$} \\
\hline $\log \left(P_{b}\right)$ & $2.17497_{-0.00098}^{+0.0011}$ & $\log$ (days) \\
\hline$\sqrt{e_{b}} \cos \omega_{b}$ & $-0.03 \pm 0.24$ & $\cdots$ \\
\hline$\sqrt{e_{b}} \sin \omega_{b}$ & $+0.34_{-0.31}^{+0.19}$ & $\cdots$ \\
\hline $\log \left(K_{b}\right)$ & $0.276_{-0.071}^{+0.061}$ & $\log \left(\mathrm{m} \mathrm{s}^{-1}\right)$ \\
\hline \multicolumn{3}{|c|}{ Model Parameters } \\
\hline$P_{b}$ & $149.61_{-0.34}^{+0.37}$ & days \\
\hline$T_{\text {conj }, b}$ & $2456670.2_{-5.6}^{+6.1}$ & $\mathrm{BJD}_{\mathrm{TDB}}$ \\
\hline$e_{b}$ & $0.19_{-0.12}^{+0.15}$ & $\cdots$ \\
\hline$\omega_{b}$ & $101_{-39}^{+69}$ & degrees \\
\hline$K_{b}$ & $1.89_{-0.28}^{+0.29}$ & $\mathrm{~m} \mathrm{~s}^{-1}$ \\
\hline$\gamma_{\text {post-upgrade Keck }}$ & $0.61 \pm 0.21$ & $\mathrm{~m} \mathrm{~s}^{-1}$ \\
\hline$\gamma_{\text {pre-upgrade Keck }}$ & $0.71_{-0.65}^{+0.64}$ & $\mathrm{~m} \mathrm{~s}^{-1}$ \\
\hline$\dot{\gamma}$ & $\equiv 0.0 \pm 0.0$ & $\mathrm{~m} \mathrm{~s}^{-1}$ day $^{-1}$ \\
\hline$\ddot{\gamma}$ & $\equiv 0.0 \pm 0.0$ & $\mathrm{~m} \mathrm{~s}^{-1}$ day $^{-2}$ \\
\hline$\sigma_{\mathrm{jitt}}$ & $2.34_{-0.12}^{+0.14}$ & $\mathrm{~m} \mathrm{~s}^{-1}$ \\
\hline
\end{tabular}

Note.

${ }^{\mathrm{a}} \mathrm{MCMC}$ jump parameters that were modified from the physical parameters in order to speed convergence and avoid biasing parameters that must physically be finite and positive.

amplitude of $2 \mathrm{~m} \mathrm{~s}^{-1}$. This would be a $M \sin i=22 M_{\oplus}$ planet orbiting just outside $1 \mathrm{au}$. The periodogram peak for this candidate falls above the eFAP threshold automatically calculated by our discovery pipeline. However, we do not believe that we have enough evidence to claim a concrete detection of a bona fide planet due to the proximity of this period to 1 year and incomplete phase coverage of the orbit. Telluric contamination of the template or problems with the barycentric correction could inject a false signal with a period near 1 year (Wright \& Eastman 2014; Fischer et al. 2016; S. Wang 2016, personal communication). The amplitude and periodicity of this signal depends on the outcomes of modeling HD $42618 \mathrm{~b}$, and MCMC runs for models including this candidate fail to converge. We will continue to monitor this star intensively with both Keck and the APF to confirm or refute this planet candidate in the upcoming years.

We find a candidate periodicity in the HD 164922 system with a period of 41.7 days and an amplitude of $1.9 \mathrm{~m} \mathrm{~s}^{-1}$. The eFAP of this 2DKLS periodogram peak is 0.00098 and falls just above our $0.1 \%$ eFAP threshold. However, we do not consider this to be a viable planet candidate due to its marginal detection and proximity to the expected rotation period for this star (44 days, Isaacson \& Fischer 2010). Further monitoring and a detailed analysis that includes the effects of rotational modulation of starspots is needed to determine the nature of this signal.

There is no evidence for significant periodic signals from other candidates in the periodograms for HD 143761. However, visually there appears to be some long-period structure in the residuals to our most probable model (see Figure 5). This marginal variability, if real, likely has a period of $\geqslant 10$ years and an amplitude of only a few $\mathrm{m} \mathrm{s}^{-1}$ and it appears to be at a shorter period than the stellar magnetic activity cycle as seen in the $S_{\mathrm{HK}}$ values for this star. Long-term
Table 4

Orbital Parameters for HD 164922

\begin{tabular}{|c|c|c|}
\hline Parameter & Value & Units \\
\hline \multicolumn{3}{|c|}{ Modified DE-MCMC Step Parameters ${ }^{\mathrm{a}}$} \\
\hline $\log \left(P_{b}\right)$ & $3.08 \pm 0.002$ & $\log$ (days) \\
\hline$\sqrt{e_{b}} \cos \omega_{b}$ & $-0.214_{-0.081}^{+0.100}$ & $\cdots$ \\
\hline$\sqrt{e_{b}} \sin \omega_{b}$ & $+0.264_{-0.130}^{+0.096}$ & $\cdots$ \\
\hline $\log \left(K_{b}\right)$ & $0.854 \pm 0.019$ & $\mathrm{~m} \mathrm{~s}^{-1}$ \\
\hline $\log \left(P_{c}\right)$ & $1.87947_{-0.00032}^{+0.00033}$ & $\log$ (days) \\
\hline$\sqrt{e_{c}} \cos \omega_{c}$ & $+0.04_{-0.30}^{+0.29}$ & $\cdots$ \\
\hline$\sqrt{e_{c}} \sin \omega_{c}$ & $+0.37_{-0.23}^{+0.14}$ & $\cdots$ \\
\hline $\log \left(K_{c}\right)$ & $0.346_{-0.062}^{+0.054}$ & $\log \left(\mathrm{m} \mathrm{s}^{-1}\right)$ \\
\hline \multicolumn{3}{|c|}{ Model Parameters } \\
\hline$P_{b}$ & $1201.1_{-5.5}^{+5.6}$ & days \\
\hline$T_{\text {conj }, b}$ & $2456778_{-19}^{+18}$ & $\mathrm{BJD}_{\mathrm{TDB}}$ \\
\hline$e_{b}$ & $0.126_{-0.050}^{+0.049}$ & $\cdots$ \\
\hline$\omega_{b}$ & $129_{-20}^{+24}$ & degrees \\
\hline$K_{b}$ & $7.15 \pm 0.31$ & $\mathrm{~m} \mathrm{~s}^{-1}$ \\
\hline$P_{c}$ & $75.765_{-0.056}^{+0.058}$ & days \\
\hline$T_{\text {conj }, c}$ & $2456277.6 \pm 2.7$ & $\mathrm{BJD}_{\mathrm{TDB}}$ \\
\hline$e_{c}$ & $0.22 \pm 0.13$ & $\cdots$ \\
\hline$\omega_{c}$ & $81_{-49}^{+45}$ & degrees \\
\hline$K_{c}$ & $2.22_{-0.29}^{+0.30}$ & $\mathrm{~m} \mathrm{~s}^{-1}$ \\
\hline$\gamma_{\text {post-upgrade Keck }}$ & $0.23 \pm 0.27$ & $\mathrm{~m} \mathrm{~s}^{-1}$ \\
\hline$\gamma_{\text {pre-upgrade Keck }}$ & $1.02 \pm 0.54$ & $\mathrm{~m} \mathrm{~s}^{-1}$ \\
\hline$\gamma_{\mathrm{APF}}$ & $0.2_{-0.48}^{+0.47}$ & $\mathrm{~m} \mathrm{~s}^{-1}$ \\
\hline$\dot{\gamma}$ & $\equiv 0.0 \pm 0.0$ & $\mathrm{~m} \mathrm{~s}^{-1}$ day $^{-1}$ \\
\hline$\ddot{\gamma}$ & $\equiv 0.0 \pm 0.0$ & $\mathrm{~m} \mathrm{~s}^{-1}$ day $^{-2}$ \\
\hline$\sigma_{\mathrm{jitt}}$ & $2.63_{-0.14}^{+0.15}$ & $\mathrm{~m} \mathrm{~s}^{-1}$ \\
\hline
\end{tabular}

Note.

${ }^{a}$ MCMC jump parameters that were modified from the physical parameters in order to speed convergence and avoid biasing parameters that must physically be finite and positive.

monitoring of this target is required to determine if this signal is real and the signature of a planetary companion.

\subsection{Chromospheric Activity}

These stars were all selected to be part of the APF-50 survey of nearby stars due, in part, to their extremely low mean chromospheric activity of $R_{\mathrm{HK}}^{\prime} \leqslant-4.95$. However, in the case of HD 42618 we do detect significant long-period variability in the $S_{\mathrm{HK}}$ values that is strongly correlated with the RVs (see Figure 8 ) that is likely the signature of the stellar magnetic activity cycle. We do not find any significant periodic signals in the $S_{\mathrm{HK}}$ values after removal of this long-period trend that might be the signature of rotation. However, we clearly identify the rotation period of the star to be 16.9 days in CoRoT photometry (see Section 5.1). We account for the activity cycle in the RV data of HD 42618 by including an additional longperiod Keplerian signal in the model.

HD 164922 shows only a linear trend in the $S_{\mathrm{HK}}$ values but we do not detect the effect of this change in chromospheric activity in the residuals to the two planet fit. There is also a very weak peak in the Lomb-Scargle periodogram $(\mathrm{L}-\mathrm{S}$, Lomb 1976; Scargle 1982) of the $S_{\mathrm{HK}}$ values at 37.8 days (see Figure 9). This may be the signature of stellar rotation 
Table 5

Orbital Parameters for HD 143761

\begin{tabular}{|c|c|c|}
\hline Parameter & Value & Units \\
\hline \multicolumn{3}{|c|}{ Modified DE-MCMC Step Parameters ${ }^{\mathrm{a}}$} \\
\hline $\log \left(P_{b}\right)$ & $1.600382 \pm 1.6 e-05$ & $\log$ (days) \\
\hline$\sqrt{e_{b}} \cos \omega_{b}$ & $+0.002 \pm 0.02$ & $\cdots$ \\
\hline$\sqrt{e_{b}} \sin \omega_{b}$ & $-0.192_{-0.010}^{+0.011}$ & $\cdots$ \\
\hline $\log \left(K_{b}\right)$ & $1.8279 \pm 0.0016$ & $\mathrm{~m} \mathrm{~s}^{-1}$ \\
\hline $\log \left(P_{c}\right)$ & $2.01091_{-0.00070}^{+0.00073}$ & $\log$ (days) \\
\hline$\sqrt{e_{c}} \cos \omega_{c}$ & $+0.01 \pm 0.19$ & $\cdots$ \\
\hline$\sqrt{e_{c}} \sin \omega_{c}$ & $-0.01 \pm 0.19$ & $\cdots$ \\
\hline $\log \left(K_{c}\right)$ & $0.573_{-0.034}^{+0.032}$ & $\log \left(\mathrm{m} \mathrm{s}^{-1}\right)$ \\
\hline \multicolumn{3}{|c|}{ Model Parameters } \\
\hline$P_{b}$ & $39.8458_{-0.0014}^{+0.0015}$ & days \\
\hline$T_{\text {conj }, b}$ & $2455759.091 \pm 0.056$ & $\mathrm{BJD}_{\mathrm{TDB}}$ \\
\hline$e_{b}$ & $0.0373_{-0.0039}^{+0.0040}$ & $\cdots$ \\
\hline$\omega_{b}$ & $270.6_{-5.8}^{+5.9}$ & degrees \\
\hline$K_{b}$ & $67.28 \pm 0.25$ & $\mathrm{~m} \mathrm{~s}^{-1}$ \\
\hline$P_{c}$ & $102.54 \pm 0.17$ & days \\
\hline$T_{\text {conj }, c}$ & $2455822 \pm 2$ & $\mathrm{BJD}_{\mathrm{TDB}}$ \\
\hline$e_{c}$ & $0.052_{-0.037}^{+0.061}$ & $\cdots$ \\
\hline$\omega_{c}$ & $190_{-140}^{+110}$ & degrees \\
\hline$K_{c}$ & $3.74 \pm 0.28$ & $\mathrm{~m} \mathrm{~s}^{-1}$ \\
\hline$\gamma_{\text {post-upgrade Keck }}$ & $-0.6 \pm 0.2$ & $\mathrm{~m} \mathrm{~s}^{-1}$ \\
\hline$\gamma_{\mathrm{APF}}$ & $-0.7_{-0.49}^{+0.50}$ & $\mathrm{~m} \mathrm{~s}^{-1}$ \\
\hline$\dot{\gamma}$ & $\equiv 0.0 \pm 0.0$ & $\mathrm{~m} \mathrm{~s}^{-1}$ day $^{-1}$ \\
\hline$\ddot{\gamma}$ & $\equiv 0.0 \pm 0.0$ & $\mathrm{~m} \mathrm{~s}^{-1} \mathrm{day}^{-2}$ \\
\hline$\sigma_{\mathrm{jitt}}$ & $2.57_{-0.13}^{+0.14}$ & $\mathrm{~m} \mathrm{~s}^{-1}$ \\
\hline
\end{tabular}

Note.

${ }^{\mathrm{a}} \mathrm{MCMC}$ jump parameters that were modified from the physical parameters in order to speed convergence and avoid biasing parameters that must physically be finite and positive.

since this is near the expected period for a star of this type and age (Isaacson \& Fischer 2010). However, this rotation period is well separated from the orbital periods of the two planets and does not influence our two planet fits.

We do not detect any long term variability in the $S_{\mathrm{HK}}$ values of HD 143761 but we see a clear peak in a periodogram of the $S_{\mathrm{HK}}$ values at 18.5 days that is likely caused by the rotational modulation of star spots (see Figure 9). Since the rotation period is well separated from the periods of either of the planets orbiting HD 143761 this does not affect our Keplerian modeling and is likely absorbed into the stellar jitter term.

\section{PHOTOMETRY}

\subsection{CoRoT Photometry of HD 42618}

HD 42618 was the target of high cadence, high precision, continuous photometric monitoring for $\approx 0.5$ years with the purpose of detecting solar like oscillations (Baglin et al. 2012). We perform a simple polynomial detrending of the space-based photometry. After removing large ramp-shaped features at the start of two long observing campaigns we then fit an 8th order polynomial to all continuous segments of the data. These segments are 2-20 days in length.
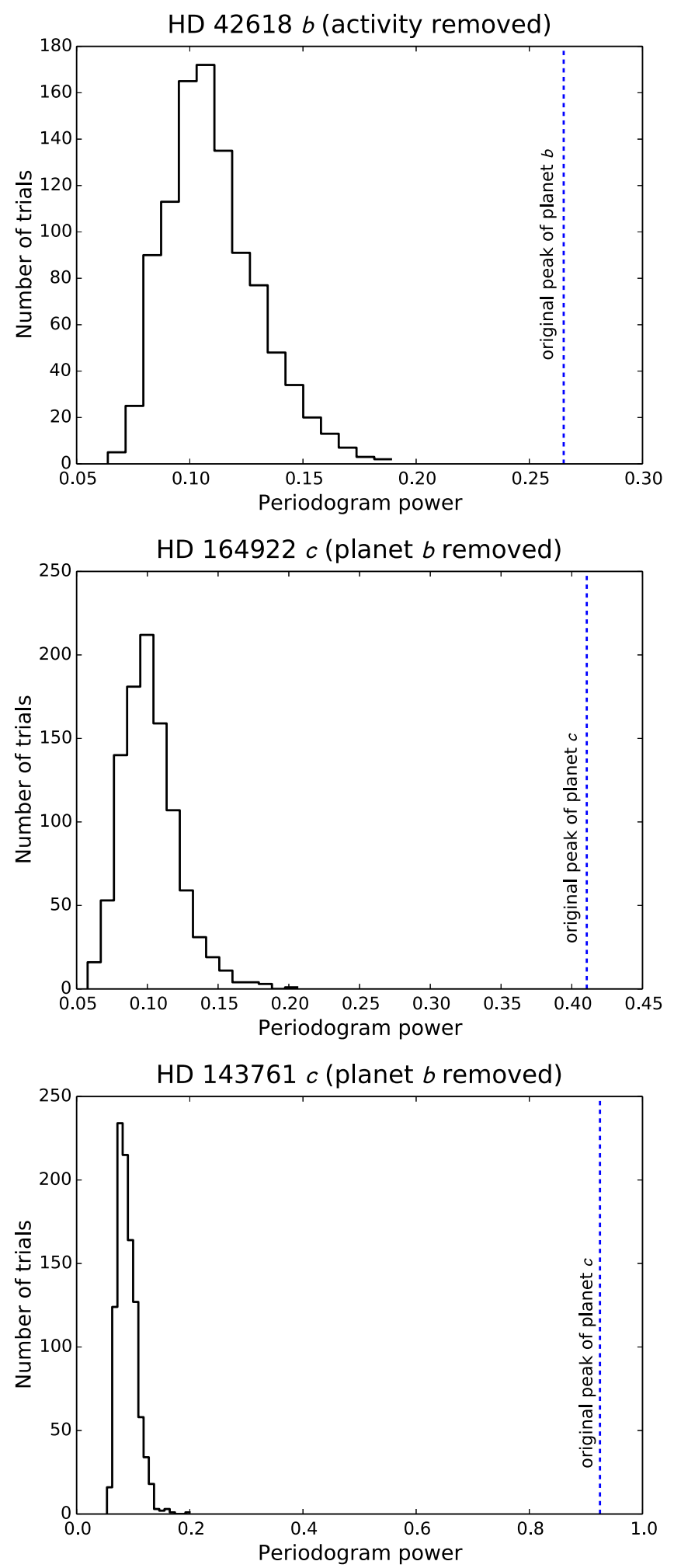

Figure 6. Graphical representation of the bootstrap false alarm tests described in Section 4.3. Top: distribution of maximum periodogram peak heights for 1000 2DKLS periodograms of scrambled RV time series for HD 42618. The long period activity signal was subtracted before scrambling the data set. The vertical dashed blue line marks the height of the original periodogram peak for planet $b$ which is clearly separated from the distribution of peaks caused by random fluctuations. Middle: same as the top panel for planet HD $164922 \mathrm{c}$. Bottom: same as the top panel for planet HD $143761 \mathrm{c}$. In each case, the scrambled RVs generate peaks with significantly lower power than the power observed from the new planetary signals. 


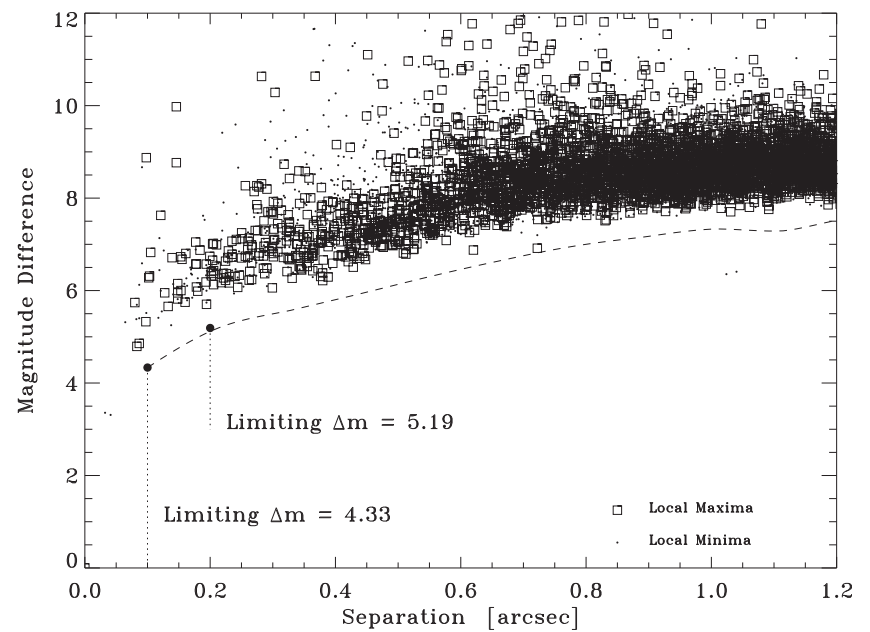

Figure 7. Limiting magnitude as a function of separation from the $\rho \mathrm{CrB}$. Also shown are a cubic spline interpolation of the $5 \sigma$ detection limit (dashed line) and limiting magnitudes for $0 . ! 1$ and $0 . " 2$.

We use the detrended photometry to look for periodic photometric variability that might be caused by rotationally modulated star spots. We detect significant variability with a period of 16.9 days but with a broad distribution of periodogram power around the highest peak (see Figure 10). This is a clear signature of stellar rotation with slightly changing phase and/or differential rotation which creates a broad distribution of increased power in Fourier space near the true rotation period. This star is very similar to the Sun in mass, age, and chemical abundance so the fact that the rotation period is also similar to that of the Sun (26 days) is not surprising. However, we note that the precise location of the highest peak in the Lomb-Scargle periodogram of HD 42618 depends on the polynomial order used to detrend the CoRoT photometry. We also tried high-pass filtering the CoRoT photometry using running median filters with window widths of 20-50 days and only analyzing continuous segments of data longer than 20 days. We found that the period of highest power is somewhat variable but always falls between $12-18$ days. Since the periodogram period is dependent on the detrending algorithm we cannot determine the rotation period of HD 42618 precisely, but we estimate that it falls within the range of 12-18 days. We do not detect any significant periodic signal in the RV data near the photometric period.

We searched through the detrended CoRoT light curve using the TERRA planet detection algorithm (Petigura et al. 2013a, 2013b). We did not find any periodic box-shaped dimmings with $\mathrm{S} / \mathrm{N}$ greater than 7. Searches of Kepler photometry commonly require $\mathrm{S} / \mathrm{N}>7$ (Jenkins et al. 2010) or $\mathrm{S} / \mathrm{N}$ $>12$ (Petigura et al. 2013b), though the $\mathrm{S} / \mathrm{N}$ threshold depends on the noise structure of the photometry. We conclude that there are no transiting planets having periods between 0.5 and 60 days with transits that are detectable above Poisson, stellar, and instrumental noise. Given the photometric noise properties of HD 42618, we can rule out planets with transits deeper than $\approx 150 \mathrm{ppm}\left(\approx 1.3 R_{\oplus}\right)$ at $\approx 5$ day orbital periods and transits deeper than $\approx 300 \mathrm{ppm}\left(\approx 1.9 R_{\oplus}\right)$ for $\approx 50$ day orbital periods. The a priori transit probability for HD $42618 \mathrm{~b}$ is only $0.8 \%$ so it is not surprising that we do not detect transits.
Table 6

Derived Planet Properties

\begin{tabular}{|c|c|c|}
\hline Parameter & Value & Units \\
\hline \multicolumn{3}{|l|}{$H D 42618$} \\
\hline$e_{b} \cos \omega_{b}$ & $-0.009_{-0.076}^{+0.06}$ & $\cdots$ \\
\hline$e_{b} \sin \omega_{b}$ & $0.14_{-0.11}^{+0.13}$ & $\cdots$ \\
\hline$a_{b}$ & $0.554 \pm 0.011$ & $\mathrm{au}$ \\
\hline$a_{b} / R_{\star}$ & $119.1_{-9.8}^{+12.0}$ & $\cdots$ \\
\hline$M_{b} \sin i_{b}$ & $14.4_{-2.4}^{+2.5}$ & $M_{\oplus}$ \\
\hline$S_{b}^{a}$ & $3.16_{-0.55}^{+0.61}$ & $S_{\oplus}$ \\
\hline$T_{\mathrm{eq}, b}{ }^{\mathrm{b}}$ & $337_{-16}^{+15}$ & $\mathrm{~K}$ \\
\hline \multicolumn{3}{|c|}{ HD 164922} \\
\hline$e_{b} \cos \omega_{b}$ & $-0.025_{-0.019}^{+0.016}$ & $\cdots$ \\
\hline$e_{b} \sin \omega_{b}$ & $0.032_{-0.022}^{+0.028}$ & $\cdots$ \\
\hline$a_{b}$ & $2.115 \pm 0.012$ & $\mathrm{au}$ \\
\hline$a_{b} / R_{\star}$ & $454.9_{-7.9}^{+8.3}$ & $\cdots$ \\
\hline$M_{b} \sin i_{b}$ & $107.6_{-4.8}^{+4.9}$ & $M_{\oplus}$ \\
\hline$S_{b}{ }^{\mathrm{a}}$ & $0.1578_{-0.0067}^{+0.0069}$ & $S_{\oplus}$ \\
\hline$T_{\mathrm{eq}, b}{ }^{\mathrm{b}}$ & $159.4 \pm 1.7$ & $\mathrm{~K}$ \\
\hline$e_{c} \cos \omega_{c}$ & $0.003_{-0.063}^{+0.073}$ & $\cdots$ \\
\hline$e_{c} \sin \omega_{c}$ & $0.079_{-0.066}^{+0.089}$ & $\cdots$ \\
\hline$a_{c}$ & $0.3351 \pm 0.0015$ & $\mathrm{au}$ \\
\hline$a_{c} / R_{\star}$ & $72.1{ }_{-1.2}^{+1.3}$ & $\cdots$ \\
\hline$M_{c} \sin i_{c}$ & $12.9 \pm 1.6$ & $M_{\oplus}$ \\
\hline$S_{c}^{\mathrm{a}}$ & $6.29_{-0.26}^{+0.27}$ & $S_{\oplus}$ \\
\hline$T_{\mathrm{eq}, c}{ }^{\mathrm{b}}$ & $400.5 \pm 4.3$ & $\mathrm{~K}$ \\
\hline \multicolumn{3}{|c|}{ HD 143761} \\
\hline$e_{b} \cos \omega_{b}$ & $7 \mathrm{e}-05_{-0.00073}^{+0.00072}$ & $\cdots$ \\
\hline$e_{b} \sin \omega_{b}$ & $-0.0072_{-0.0012}^{+0.0011}$ & $\cdots$ \\
\hline$a_{b}$ & $0.2196_{-0.0025}^{+0.0024}$ & $\mathrm{au}$ \\
\hline$a_{b} / R_{\star}$ & $34.66_{-0.76}^{+0.78}$ & $\cdots$ \\
\hline$M_{b} \sin i_{b}$ & $332.1_{-7.6}^{+7.5}$ & $M_{\oplus}$ \\
\hline$S_{b}^{\mathrm{a}}$ & $34.7_{-2.0}^{+2.1}$ & $S_{\oplus}$ \\
\hline$T_{\mathrm{eq}, b} \mathrm{~b}$ & $614.0_{-9.0}^{+9.1}$ & $\mathrm{~K}$ \\
\hline$e_{c} \cos \omega_{c}$ & $0.0001_{-0.011}^{+0.013}$ & $\cdots$ \\
\hline$e_{c} \sin \omega_{c}$ & $-0.0001_{-0.015}^{+0.011}$ & $\cdots$ \\
\hline$a_{c}$ & $0.4123_{-0.0047}^{+0.0046}$ & $\mathrm{au}$ \\
\hline$a_{c} / R_{\star}$ & $65.1_{-1.4}^{+1.5}$ & $\cdots$ \\
\hline$M_{c} \sin i_{c}$ & $25 \pm 2$ & $M_{\oplus}$ \\
\hline$S_{c}^{\mathrm{a}}$ & $9.85_{-0.56}^{+0.6}$ & $S_{\oplus}$ \\
\hline$T_{\mathrm{eq}, c}{ }^{\mathrm{b}}$ & $448.1 \pm 6.6$ & $\mathrm{~K}$ \\
\hline
\end{tabular}

Notes.

a Stellar irradiance received at the planet relative to the Earth.

b Assuming a bond albedo of 0.32 (Demory 2014).

\subsubsection{Asteroseismic Mass Determination}

Convection in the outer layers of a star excites stochastic oscillations, which can be observed on the stellar surface. In the case of main sequence stars, these oscillations manifest themselves as periodic variations on the order of $\mathrm{cm} \mathrm{s}^{-1}$ in radial velocity data or $\sim$ ppm in photometric data. Photometric space telescopes such as CoRoT proved to be quite effective for measuring and characterizing these oscillations, which can be used to derive global stellar properties (such as radius, mass and age), as well as to constrain the stellar interior (e.g., Michel et al. 2008; Chaplin \& Miglio 2013). 

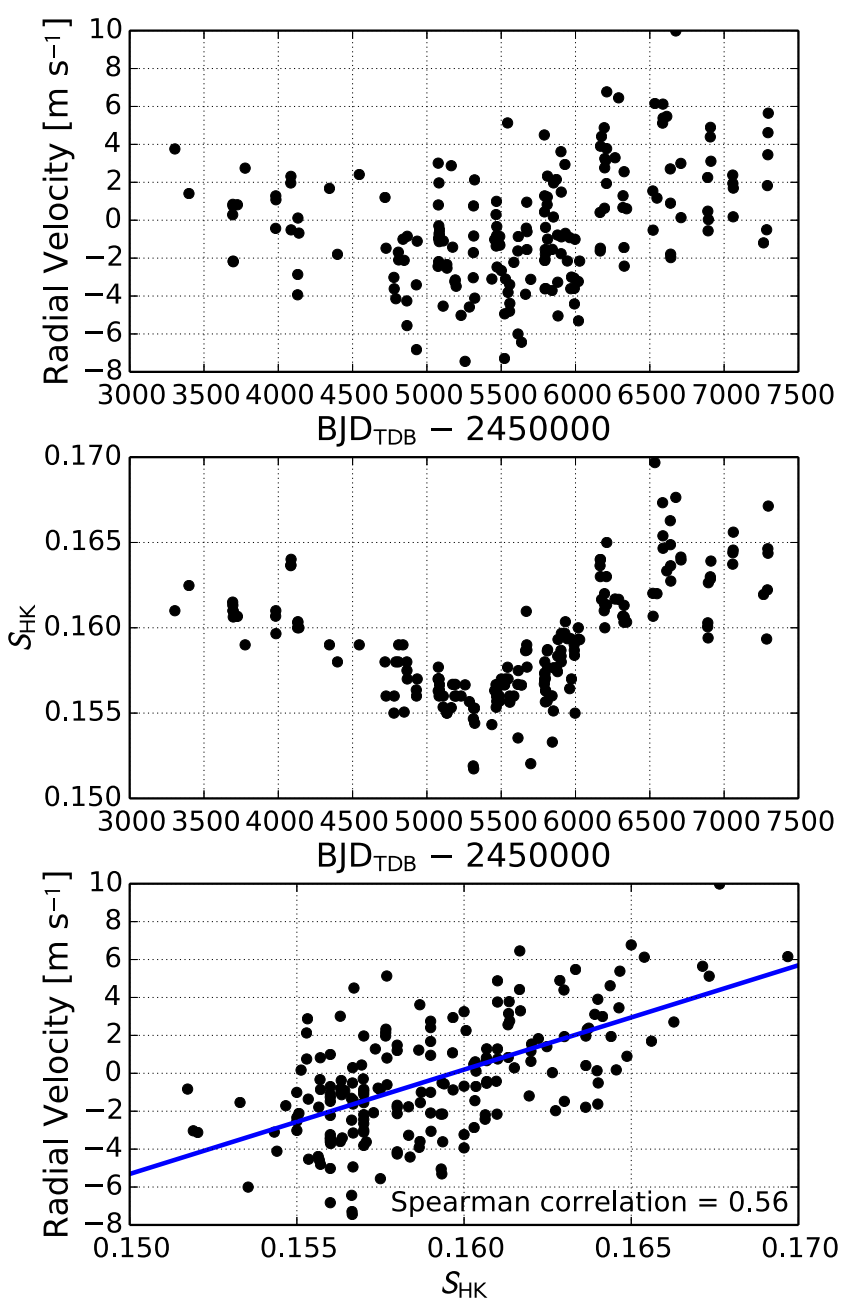

Figure 8. Velocity-activity correlation for HD 42618. A discussion of the chromospheric activity of each of the three stars can be found in Section 4.6. Top: binned RV time series of the post-upgrade Keck data with planet b. Middle: binned $S_{\mathrm{HK}}$ time series of the post-upgrade Keck data only. Note the similarities between the variability in the top and middle panels. Bottom: spearman rank correlation test of the velocities with $S_{\mathrm{HK}}$ values (Spearman 1904). We do not subtract this correlation from the RVs of HD 42618 but instead model the magnetic activity cycle as an additional long-period Keplerian (see Section 4.2).

We measured the mass of HD42618 from CoRoT photometry obtained during two long observing runs spanning 79 and 94 days, respectively. Through a Fourier analysis of the CoRoT lightcurve, we produced the power spectral density function shown in Figure 12. We then stacked the power spectrum in equally sized pieces to create an echelle diagram, revealing the distinct $l=0,1$, and 2 latitudinal modes of oscillation. We then collapsed this echelle diagram, effectively creating a binned power spectrum, and fit the power excess with a Gaussian to measure a maximum oscillation power frequency $\nu_{\max }$ of $3.16 \pm 0.10 \mathrm{mHz}$. We then collapsed the echelle diagram along the perpendicular axis to preserve the frequency spacing, computed its autocorrelation, and fit the autocorrelation with a Gaussian to measure a large oscillation frequency spacing $\Delta \nu$ of $141.6 \pm 0.8 \mu \mathrm{Hz}$. Using scaling relations (Christensen-Dalsgaard \& Frandsen 1983; Kjeldsen \& Bedding 1995; Kallinger et al. 2010; Huber et al. 2011), solar parameters taken from Huber et al. (2011), and an effective temperature equivalent to the Sun's within errors (Morel et al.
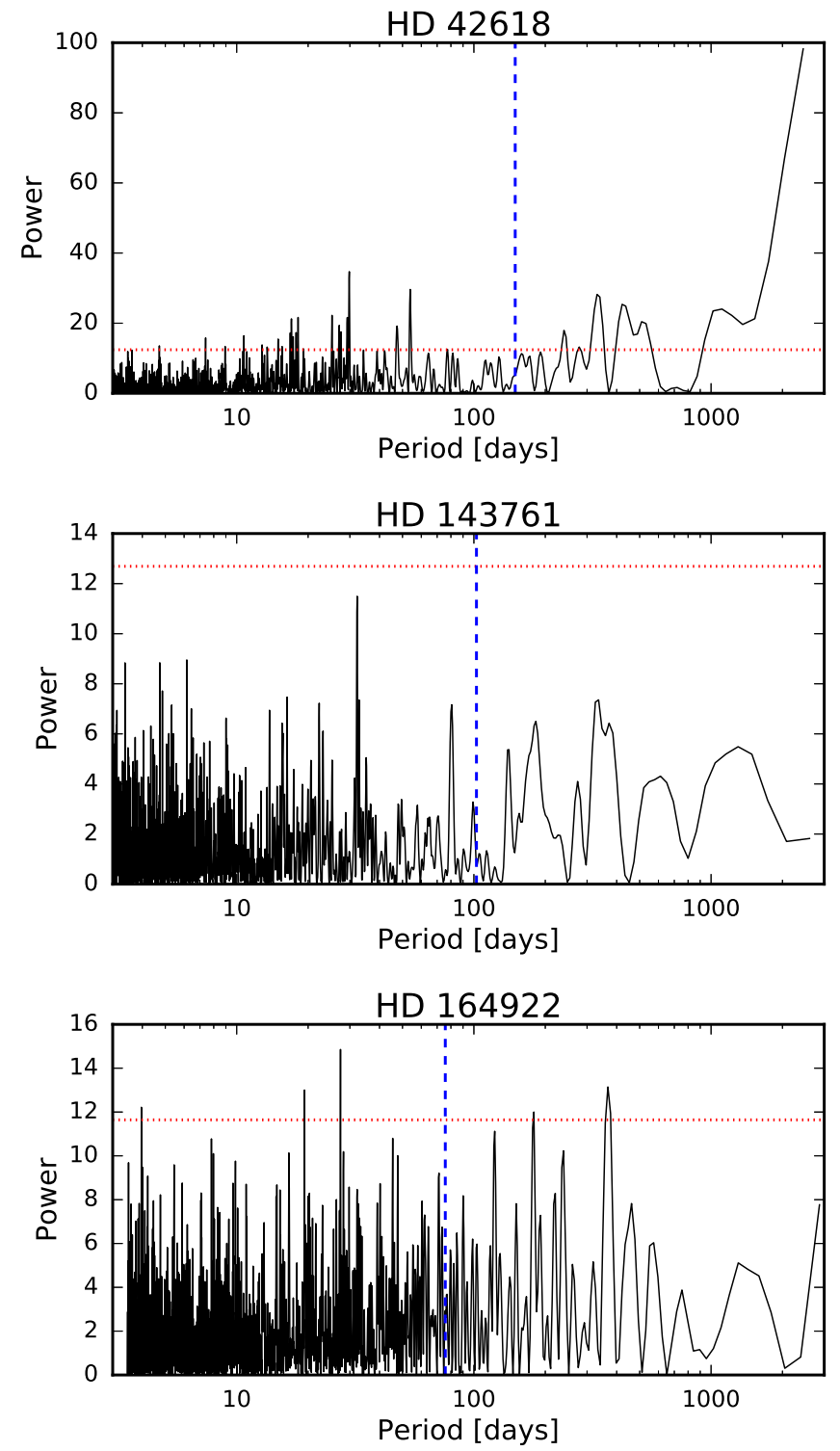

Figure 9. Lomb-Scargle periodograms of $S_{\mathrm{HK}}$ chromospheric activity. In each panel the period of the planet announced in this work is marked by the blue dashed line and the power corresponding to an analytical false alarm probability of $1 \%$ is marked by the red dotted line (Schwarzenberg-Czerny 1998). $S_{\mathrm{HK}}$ values measured from spectra with $\mathrm{S} / \mathrm{N}<40$ per pixel or exposure times $>25 \%$ longer then the median exposure time (due to clouds and/or seeing) can be badly contaminated by the solar spectrum and cause our $S_{\mathrm{HK}}$ extraction pipeline to produce large outliers. These measurements were excluded before calculating the periodograms. No significant periodicity is detected in any of the stars at the orbital periods of the new planets. Top: periodogram of $S_{\mathrm{HK}}$ values for HD 42618. Middle: periodogram of $S_{\mathrm{HK}}$ values for HD 143761. Bottom: periodogram of $S_{\mathrm{HK}}$ values for HD 164922.

2013) we measure an asteroseismic radius of $0.95 \pm 0.05 R_{\odot}$, and an asteroseismic mass of $0.93 \pm 0.13 M_{\odot}$. This is in agreement with a previous CoRoT asteroseismic analysis (Barban et al. 2013) and our estimate of the stellar mass and radius of HD42618 using our spectroscopic constraints and the Torres et al. (2010) relations. The precision on the asteroseismic mass is lower compared to our spectroscopic + isochrone mass but it is much less model-dependent. If we were to fit the spectroscopic parameters to isochrones derived using different input physics we may find that the error on the spectroscopic mass is much larger. We adopt the higher precision, 

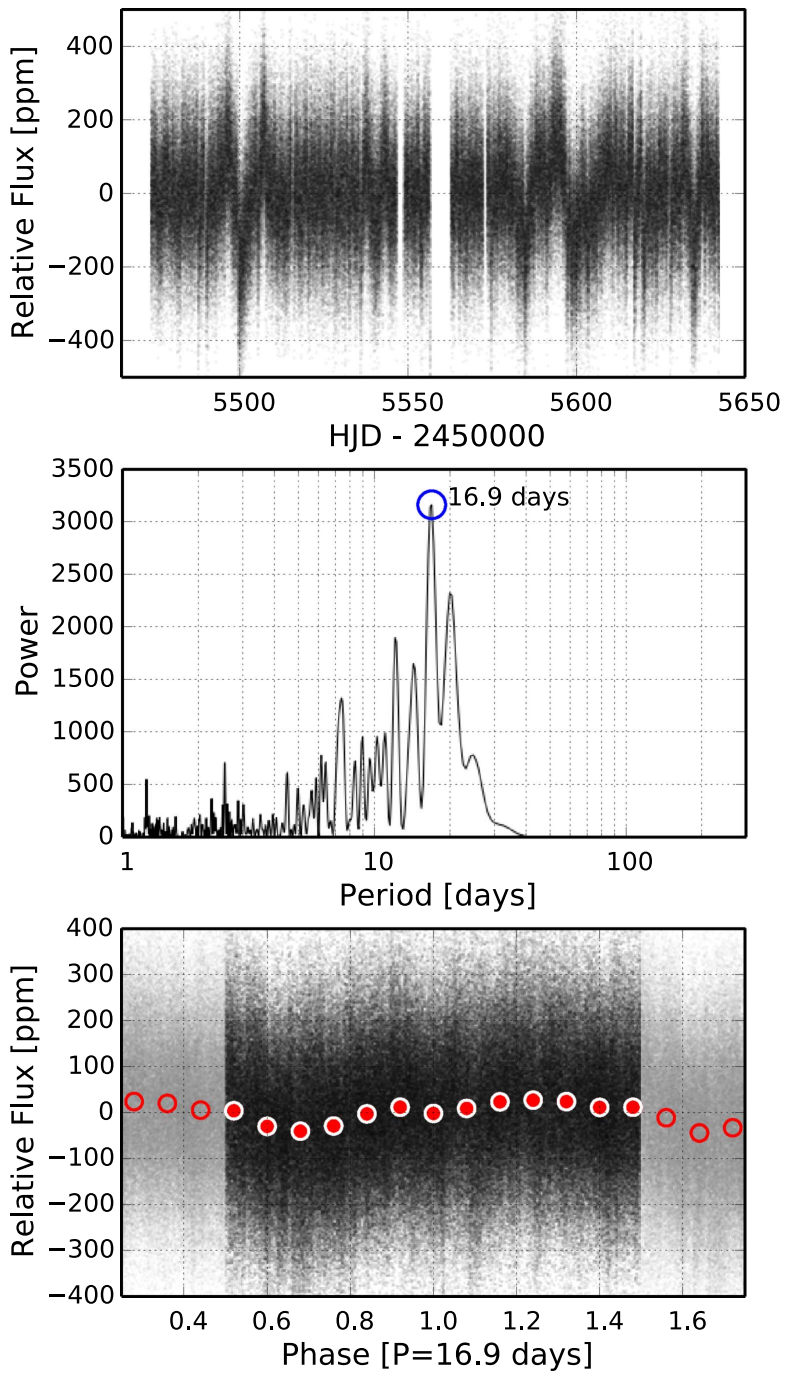

Figure 10. CoRoT photometry of HD 42618 discussed in Section 5.1. Top detrended light curve. Middle: Lomb-Scargle periodogram of the light curve. Bottom: photometry phase-folded to the period corresponding to the highest peak in the Lomb-Scargle periodogram (16.9 days). We also bin the photometry with bin widths of 0.05 units of phase (red circles).

spectroscopic mass for all calculations of planet minimum masses and orbital separations.

\subsection{Automated Photometric Telescope (APT) Photometry}

Long-term photometric observations of HD 42618, HD 143761, and HD 164922 were collected with Tennessee State University's T11 $0.80 \mathrm{~m}$, T4 $0.75 \mathrm{~m}$, and T12 $0.80 \mathrm{~m}$ APTs at Fairborn Observatory. These three stars are among a collection of more than 300 being observed by the APTs to study magnetic cycles in solar-type stars (e.g., Lockwood et al. (2013) and references therein) and have APT observational histories between 15 and 23 years. At the beginning of the APF survey, the vast majority of the target stars were already being observed by the APTs. The remaining few have been added so that all 51 stars in the APF survey are also being observed nightly by the APTs.

The APTs are equipped with two-channel precision photometers that use a dichroic filter and two EMI 9124QB bi-alkali photomultiplier tubes to measure the Strömgren $b$ and $y$ pass bands simultaneously. The APTs are programmed to make
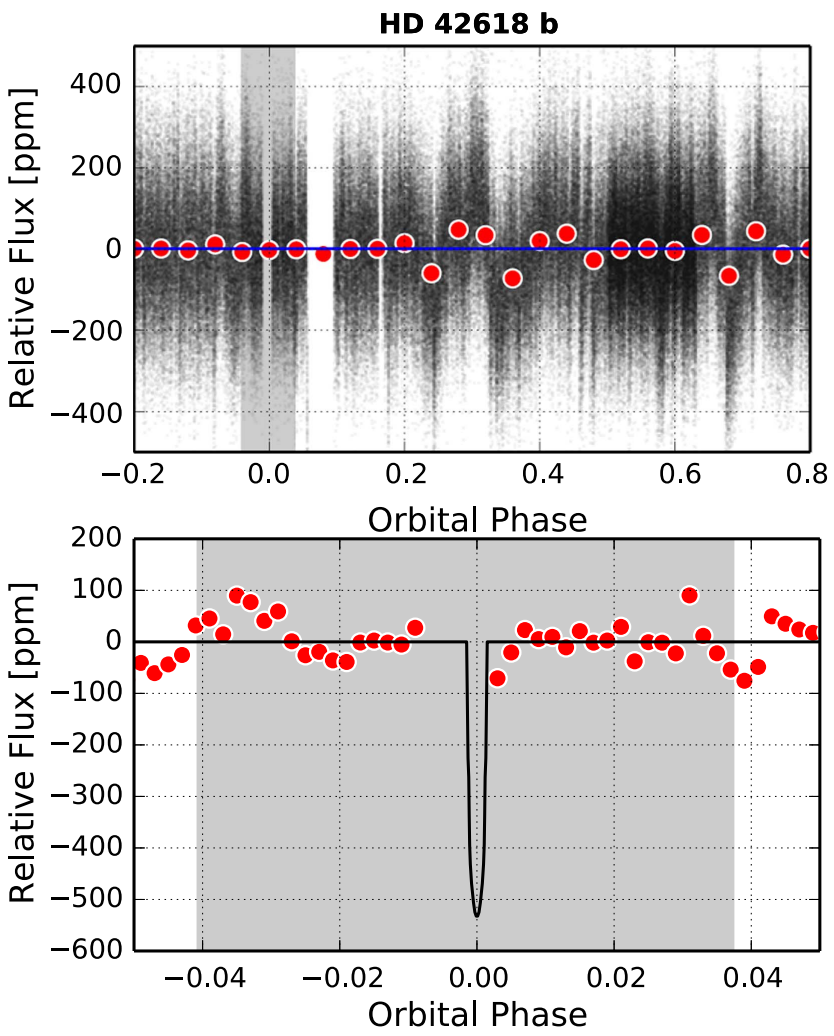

Figure 11. CoRoT photometry of HD 42618 phase-folded to the orbital period of planet b. The transit search for HD $42618 \mathrm{~b}$ is discussed in Section 5.1. Top: photometry over the full orbital phase of planet $b$. The red circles are binned photometric measurements with bin widths of 0.04 units of orbital phase. The gray shaded region shows the $1 \sigma$ uncertainty on the time of inferior conjunction derived from the RV modeling. Bottom: same as top panel with the $\mathrm{x}$-axis zoomed-in near the time of inferior conjunction. In this panel we only plot the measurements binned with bin widths of 0.002 units of orbital phase. Again, the shaded region represents the $1 \sigma$ uncertainty on the time of inferior conjunction. The black transit model shows the predicted transit depth for a solid iron planet using the mass-radius relation of Weiss \& Marcy (2014).

differential brightness measurements of a program star with respect to three comparison stars. For the APF project, we use the two best comparison stars $(\mathrm{C} 1$ and $\mathrm{C} 2)$ and compute the differential magnitudes $P-C 1, P-C 2$, and $C 2-C 1$, correct them for atmospheric extinction, and transform them to the Strömgren system. To maximize the precision of the nightly observations, we combine the differential $b$ and $y$ observations into a single $(b+y) / 2$ "passband" and also compute the differential magnitudes of the program star against the mean brightness of the two comparison stars. The resulting precision of the individual $P-(C 1+C 2) / 2_{b y}$ differential magnitudes ranges between $\sim 0.0010 \mathrm{mag}$ and $\sim 0.0015$ mag on good nights. Further details of our automatic telescopes, precision photometers, and observing and data reduction procedures can be found in Henry (1999), Eaton et al. (2003), and Henry et al. (2013).

\subsubsection{APT Photometry of HD 42618}

We collected 2241 relative flux measurements of HD 42618 over the past 15 years. We search for photometric variability on short timescales by first subtracting the mean magnitude from each observing season to remove seasonal offsets. This removes all astrophysical and systematic instrumental variability on timescales longer then one year. A L-S period search 


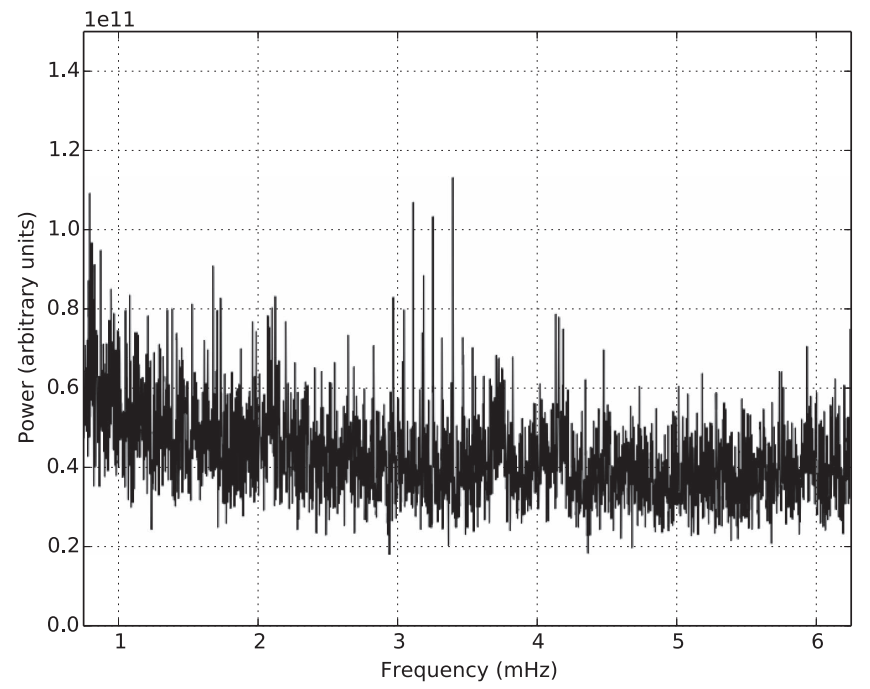

Figure 12. Smoothed one-dimensional power spectrum of HD 42618 from the CoRoT data. The comb of peaks in the power spectrum near a frequency of 3 $\mathrm{mHz}$ is the signature of solar-like asteroseismic oscillations. Our asteroseismic analysis of HD 42618 is described in Section 5.1.1.

returns a very weak periodicity with a period of 16.5 days and an amplitude of $0.3 \mathrm{mmag}$. This may be the same signature of stellar rotation as detected in the CoRoT data but it is too close to the precision limit of the ground-based dataset to be certain.

Photometric variability on the timescale of the orbital period may indicate that the RV fluctuations are the result of rotational modulation of star spots (Queloz et al. 2001). We find no evidence of photometric variability at the orbital period of the planet to the limit of our photometric precision. A least-squares sine fit on the orbital period of HD 42618 gives a semiamplitude of just $0.000037 \mathrm{mag}$, showing the complete absence of any surface activity that could affect the radial velocities. Figure 13 shows the full photometric dataset and Figure 14 shows the photometry phase folded to the orbital period of planet $b$. The lack of variability at the orbital period is consistent with the results of the CoRoT analysis and strengthens our claim that the RV fluctuations are caused by a Neptune-mass planet orbiting HD 42618. We also find no evidence of the transit of HD $42618 \mathrm{~b}$ in the APT data.

Figure 15 shows the mean $S_{\mathrm{HK}}$ values from Keck and APF and the long-term photometric variability of HD 42618 by plotting the seasonal means of both the $S$ values and APT photometry. For old solar type stars we expect a positive correlation of chromospheric activity as measured by the $S_{\mathrm{HK}}$ values with the mean brightness since the number of bright faculae regions on the star increases during more active periods. However, in this case we see no correlation of mean brightness with $S_{\mathrm{HK}}$. Young stars typically show a negative correlation of brightness with $S_{\mathrm{HK}}$ because their photometric variations are spot-dominated instead of faculae dominated. While somewhat unusual, this behavior is not unprecedented among similar stars (Hall et al. 2009).

\subsubsection{APT Photometry of HD 164922}

We collected a total of 1095 photometric measurements for HD 164922 over the past 11 observing seasons from 2005 to 2015. As with HD 42618 we remove seasonal offsets from the photometry to search for short period variability and search for transits of HD164922 b and c. We find no significant periodic variability with a period between 1 and 100 days and do not
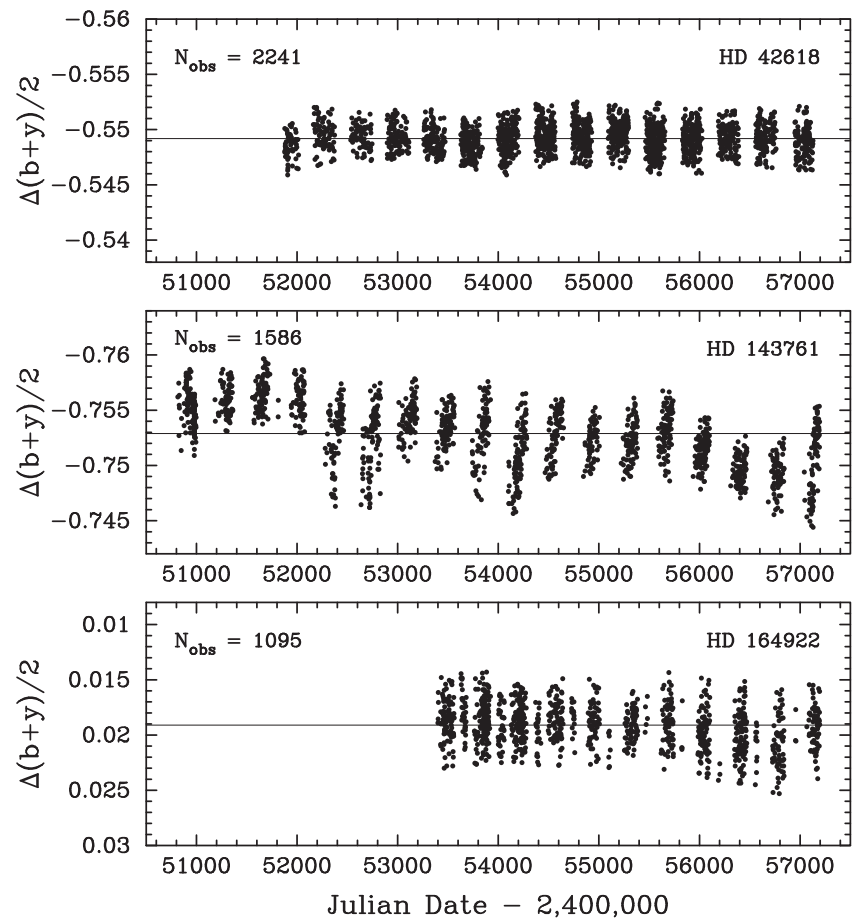

Figure 13. Long-term photometric observations of the planetary candidate host stars HD 42618 (top), HD 143761 (middle), and HD 164922 (bottom) acquired with TSU's T11 $0.80 \mathrm{~m}$, T4 $0.75 \mathrm{~m}$, and T12 $0.80 \mathrm{~m}$ APTs at Fairborn Observatory in southern Arizona. All three stars are plotted with identical $\mathrm{x}$ and y scales. The horizonal line in each panel marks the mean of each data set. The APT photometry and analysis are described in Section 5.2.
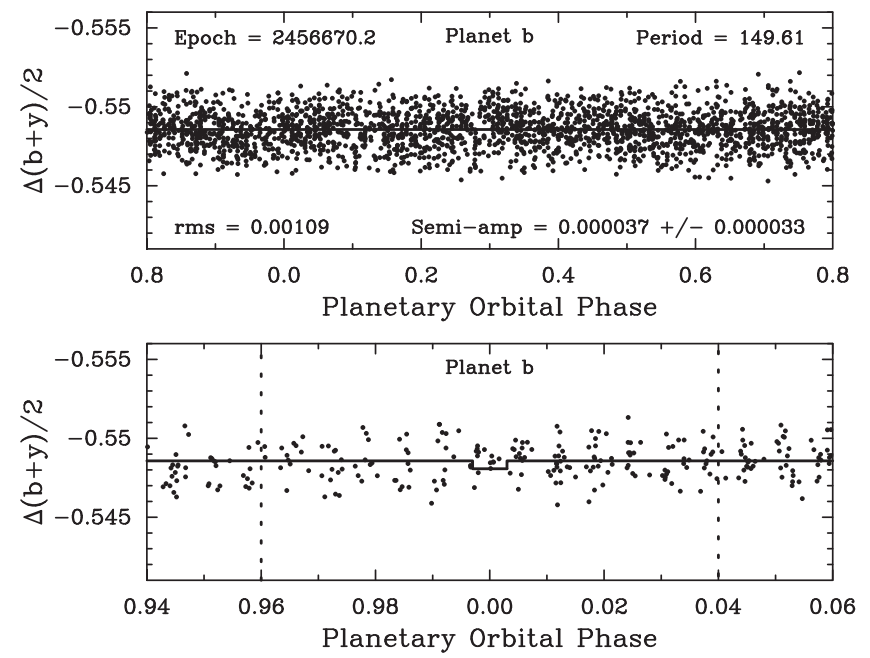

Figure 14. Top: fifteen years of photometric observations of HD 42618 from the top panel of Figure 13 plotted against the 149.6 day planetary orbital period and time of conjunction derived from the radial velocity observations. A leastsquares sine fit on the radial velocity period gives a semi-amplitude of just $0.000037 \pm 0.000033 \mathrm{mag}$, firmly establishing the lack of stellar activity on the radial velocity period and thus confirming the presence of stellar reflex motion caused by an orbiting planet. Bottom: closeup of the observations near the time of planetary conjunction at phase 0.0 . The solid line shows a toy model transit of a sphere of constant $1.0 \mathrm{~g} \mathrm{~cm}^{3}$ density and radius determined by the relation of Weiss \& Marcy (2014). The vertical lines mark the uncertainty in the predicted transit times. Our current photometric observations provide no evidence for transits.

detect the rotation period of the star. We also find no evidence of transits for either planet $\mathrm{b}$ or $\mathrm{c}$ or periodic photometric variability at the orbital period of either planet (Figure 16). 


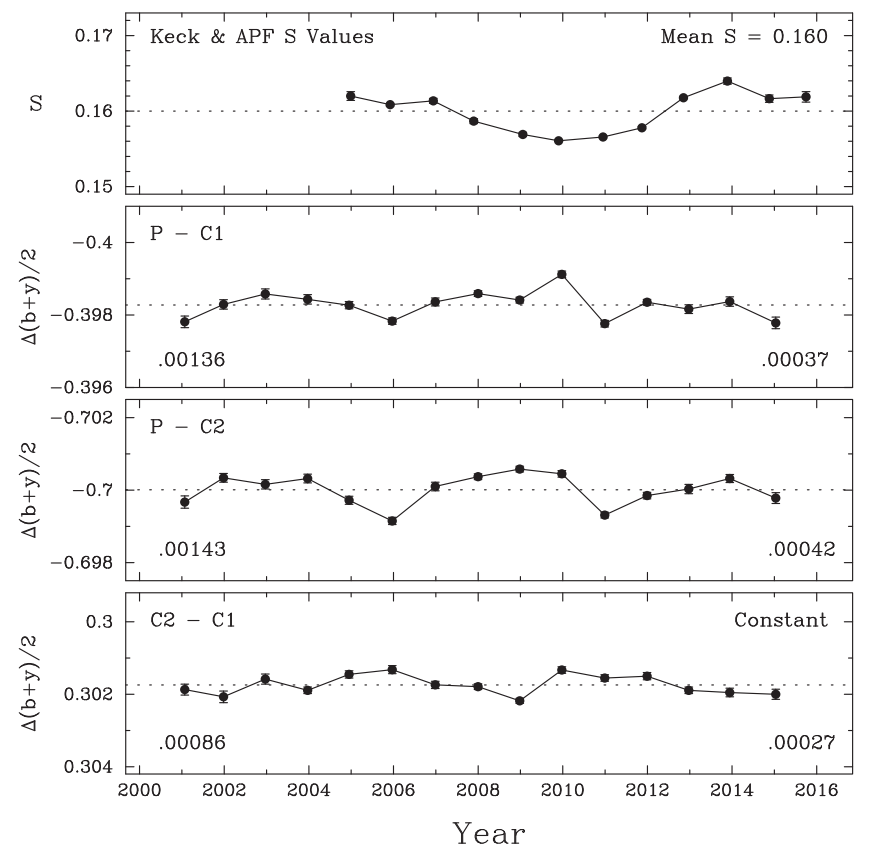

Figure 15. Top: yearly means of the Mt. Wilson-calibrated $S$ values acquired along with the radial velocity measurements. Bottom three panels: yearly means of HD 42618's $P-C 1, P-C 2$, and $C 2-C 1$ differential magnitudes. The horizontal dotted lines designate the grand means of the observations while the numbers in the lower-left and lower-right give the total range and standard deviation of each data set, respectively. It is evident that we have resolved low-level brightness variability in HD 42618 compared to the two comparison stars, $C 1$ and $C 2$. Low-amplitude cycles of roughly $0.001 \mathrm{mag}$ over 5 years are seen in both the $P-C 1$ and $P-C 2$ light curves. There appears to be little or no correlation of $S$ values with photometric brightness.

We study the long-term photometric variability of $\mathrm{HD}$ 164922 by comparing the mean brightness of the star to the $S_{\mathrm{HK}}$ activity index (Figure 17). In contrast to the results for HD 42618 , in this case we see a clear positive correlation of the brightness of HD 164922 with the $S_{\mathrm{HK}}$ index. It is interesting that we do not see a RV versus $S_{\mathrm{HK}}$ correlation for HD 164922, but we do find that the RV is strongly correlated with $S_{\mathrm{HK}}$ for HD 42618 where the photometry is not. In other cases we have seen a correlation in both the photometry and RV data (e.g., Fulton et al. 2015).

\subsubsection{APT Photometry of HD 143761}

We collected 1586 photometric measurements of HD 143761 over the past 18 observing seasons from 1997 to 2015 (Figure 13). Our reduction and analysis techniques are the same as for HD 42618 and HD 164922 discussed in the previous two sections. We find no evidence of the photometric signature of rotationally modulated star spots or photometric variability at the orbital periods of HD $143761 \mathrm{~b}$ or $\mathrm{c}$. There is no evidence of transits of either planet $b$ or c (Figure 18), however shallow transits of a rocky planet $\mathrm{c}$ can not be ruled out by this dataset.

The mean photometric brightness binned by observing season is well correlated with the $S_{\mathrm{HK}}$ values measured using Keck and APF as expected for an old solar type star. As with HD 164922, we do not see a correlation of $S_{\mathrm{HK}}$ with RV but there is a positive correlation of $S_{\mathrm{HK}}$ with mean brightness (Figure 19).

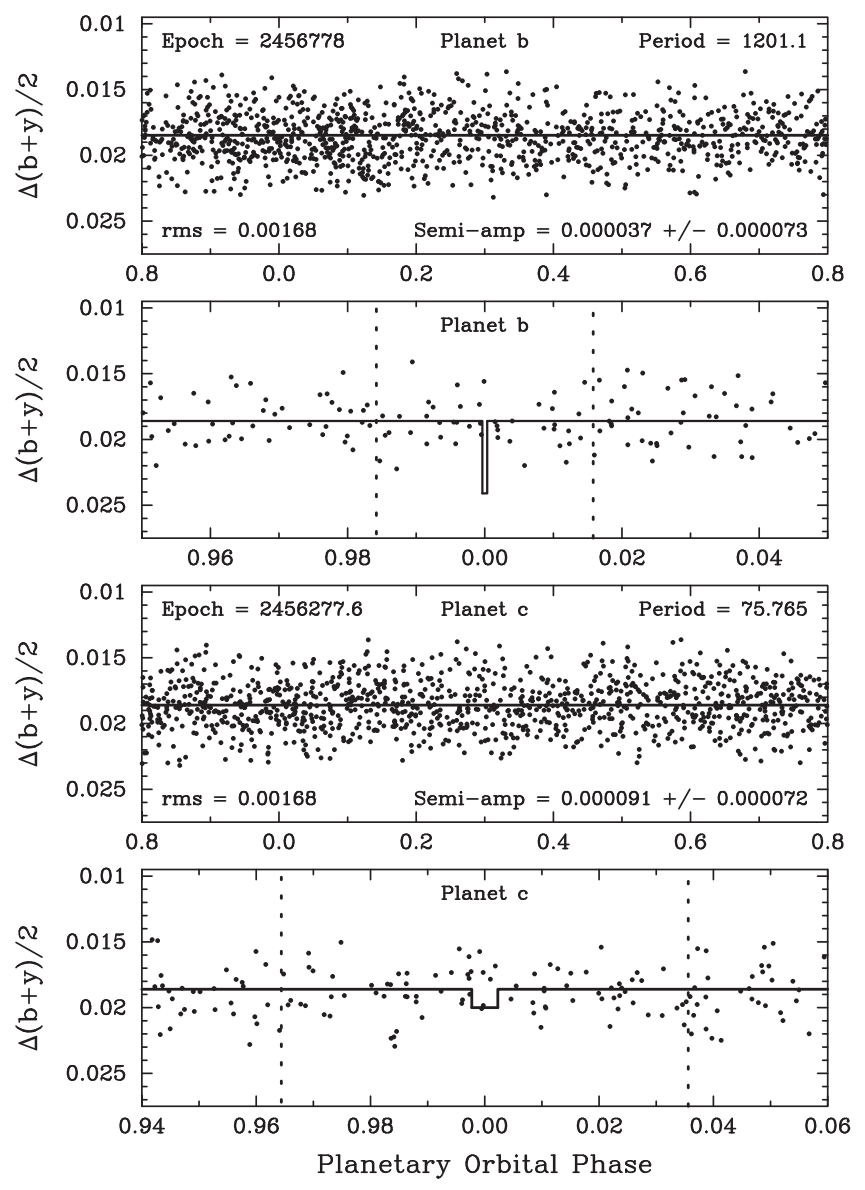

Figure 16. Same as Figure 14 but for HD 164922 b and c.

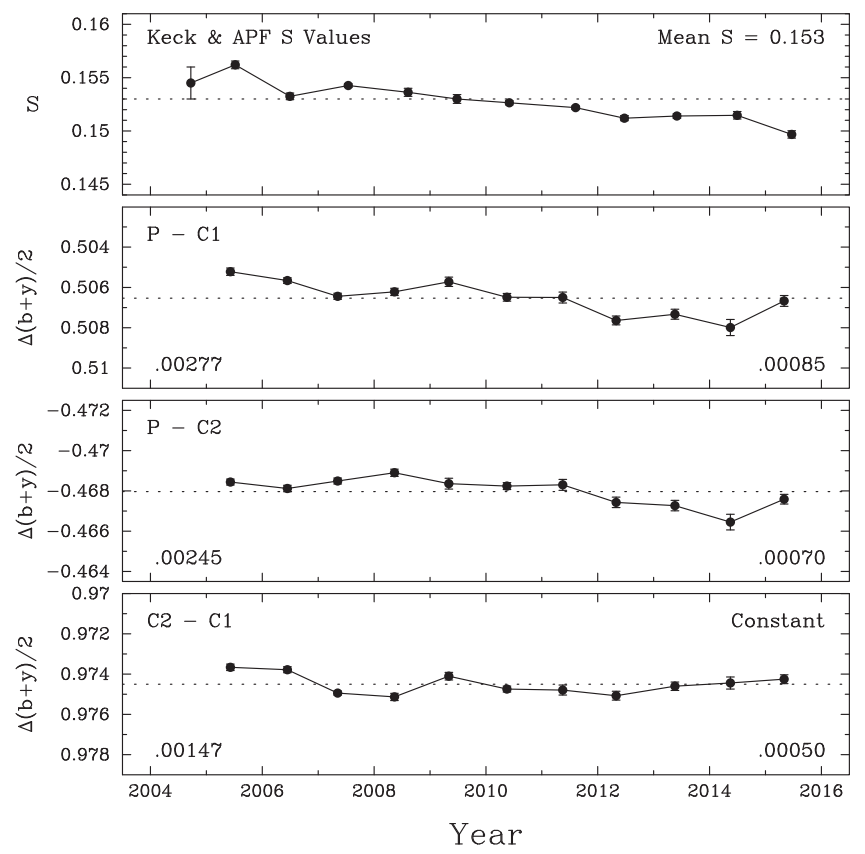

Figure 17. Same as Figure 15 but for HD 164922. In this case we see a positive correlation of the brightness of HD 164922 with the $S_{\mathrm{HK}}$ index.

\section{DISCUSSION AND SUMMARY}

We present the discovery of three approximately Neptune mass planets orbiting three bright, nearby stars. The planet 

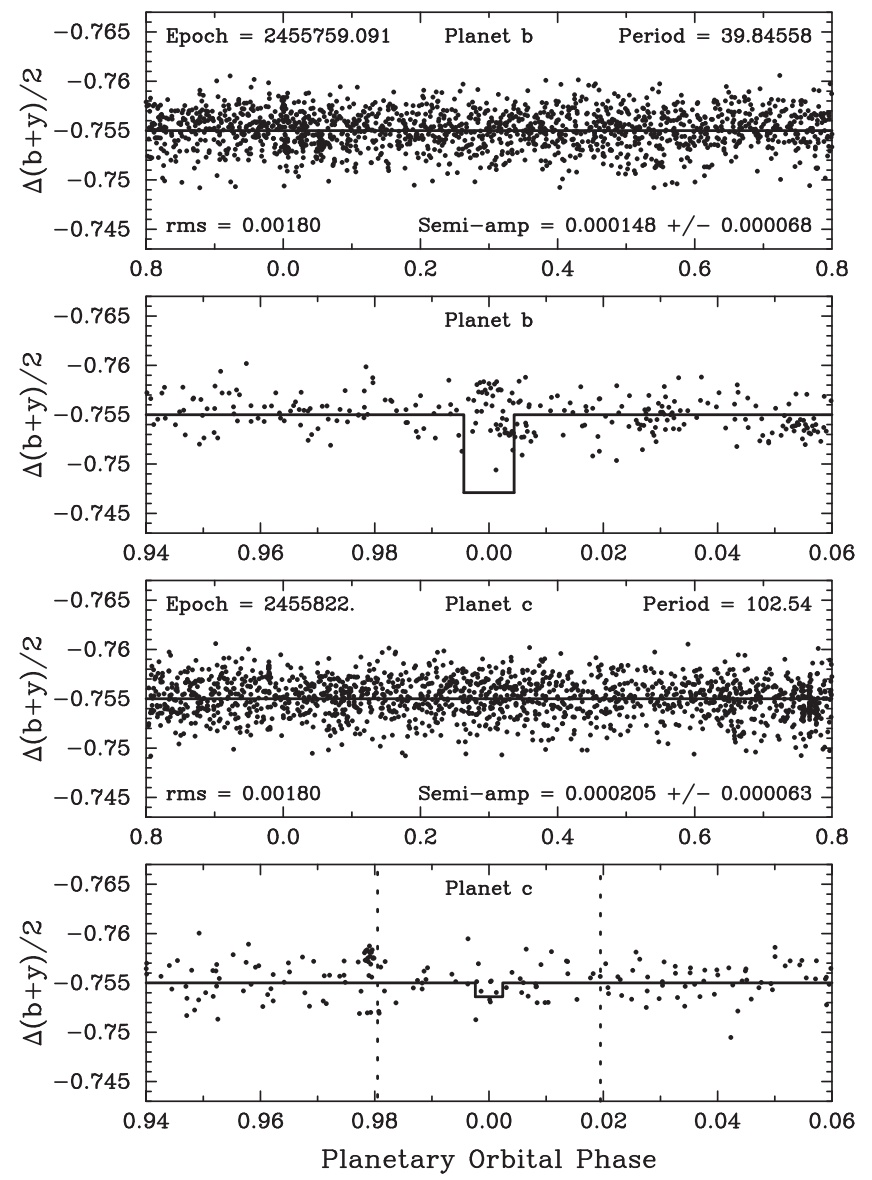

Figure 18. Same as Figure 14 but for HD $143761 \mathrm{~b}$ and c.
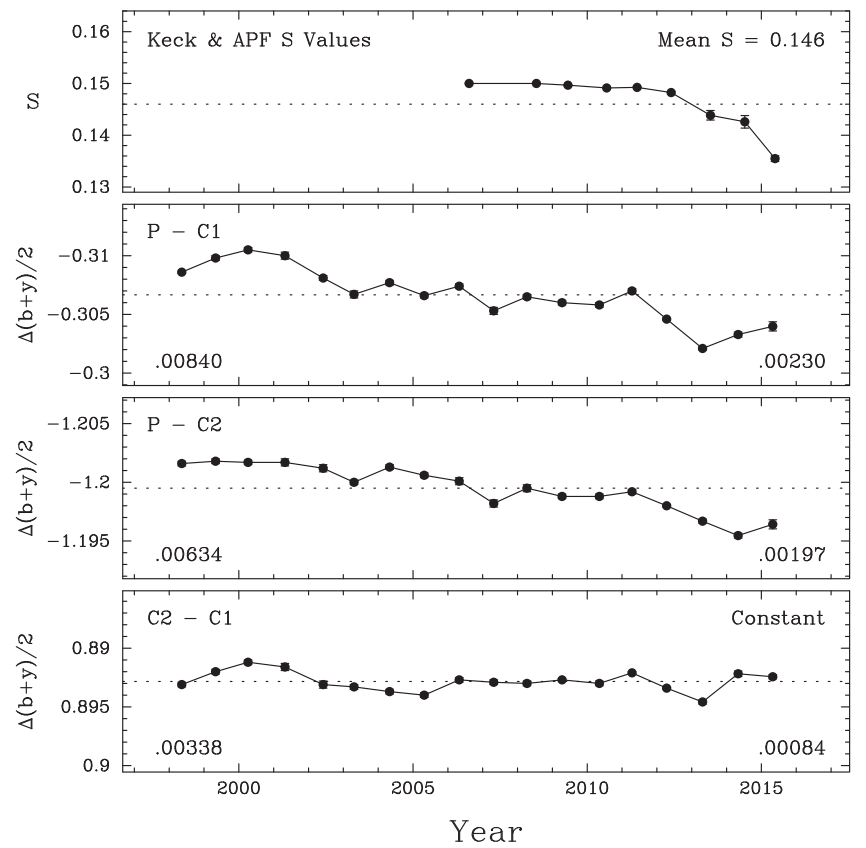

Figure 19. Same as Figure 15 but for HD 143761. In this case we again see a positive correlation of the brightness of HD 143761 with the $S_{\mathrm{HK}}$ index.

orbiting HD 42618 has a minimum mass of $M \sin i=15.4 \pm 2.4 M_{\oplus}$ and is the first discovered to orbit this star. There has been some discussion in the literature that stellar abundance patterns similar to the Sun might be evidence of the formation of terrestrial planets similar to those that exist in our solar system (e.g., Meléndez et al. 2009; González Hernández et al. 2010). While we can not rule out the existence of terrestrial planets in the HD 42618 system, the presence of a temperate Neptune mass planet orbiting at 0.554 au with an orbital period of 149 days shows that, at the present time, this system is not a close analogue to our own solar system. We cannot determine the initial planetary architecture of this system because migration may have played an important role to sculpt the current configuration.

We also detect the signature of the stellar magnetic activity cycle with a period of $\sim 12$ years. This activity cycle manifests as a $3.1 \mathrm{~m} \mathrm{~s}^{-1}$ amplitude signal in the RV time series. We identify the rotation period of the star to be $\approx 17$ days using public data from the CoRoT space telescope. Transits of HD $42618 \mathrm{~b}$ are expected to be extremely unlikely and we do not find any evidence for transits of this planet in the CoRoT data. This is a temperate planet receiving only 3.1 times the radiation that the Earth receives from the Sun. The planet's equilibrium temperature, assuming a bond albedo of 0.32 (Demory 2014), is $337 \mathrm{~K}$. We perform an asteroseismic study of HD 42618 to detect solar like oscillations and measure a precise stellar radius and mass.

HD $164922 \mathrm{c}$ is the second planet in a system previously known to host one Jupiter mass planet orbiting at $2.1 \mathrm{au}$. The new planet announced in this work is a sub-Neptune mass planet with $M \sin i=12.9 \pm 1.6 M_{\oplus}$ orbiting at a distance of $a=0.34$ au and an orbital period of 75 days. This planet is also temperate with an equilibrium temperature of $401 \mathrm{~K}$ and receiving 6.3 times the flux received by the Earth from the Sun.

HD $143761 \mathrm{c}$ is the second planet in a system previously known to host a warm Jupiter mass planet orbiting with a period of 39 days. The new planet is a super-Neptune with $M \sin i=25 \pm 2 M_{\oplus}$ orbiting with a period of 102 days. This planet is the warmest of the three with a stellar irradiance 9.6 times that of the Earth-Sun system and an equilibrium temperature of $445 \mathrm{~K}$. We find that the previous low inclination orbit for HD 143761 detected in Hipparcos astrometry can not be stable with the presence of HD $143761 \mathrm{c}$.

These three planets are some of the nearest long period Neptune mass planets yet discovered. They demonstrate the capabilities of the combined Keck+APF-50 survey and are the beginning of a complete census of small planets in the local neighborhood.

We thank the many observers who contributed to the measurements reported here. We thank Kyle Lanclos, Matt Radovan, Will Deich and the rest of the UCO Lick staff for their invaluable help shepherding, planning, and executing observations, in addition to writing the low-level software that made the automated APF observations possible. We thank Gail Schaefer for her help with the calculations related to the CHARA interferometric observations. We thank Debra Fischer, Jason Wright, and John Johnson for their many nights of observing that contributed to the Keck data presented in this work. We gratefully acknowledge the efforts and dedication of the Keck Observatory staff, especially Scott Dahm, Greg Doppman, Hien Tran, and Grant Hill for support of HIRES and Greg Wirth for support of remote observing. We are grateful to the time assignment committees of the University of Hawai' $i$, the University of California, and NASA for their generous allocations of observing time. Without their long-term commitment to 
$\mathrm{RV}$ monitoring, these planets would likely remain unknown. We acknowledge R Paul Butler and SS Vogt for many years of contributing to the data presented here. AWH acknowledges NSF grant AST-1517655 and NASA grant NNX12AJ23G. LMW gratefully acknowledges support from Ken and Gloria Levy. DH acknowledges support by the Australian Research Council's Discovery Projects funding scheme (project number DE140101364) and support by the National Aeronautics and Space Administration under Grant NNX14AB92G issued through the Kepler Participating Scientist Program. This material is based upon work supported by the National Science Foundation Graduate Research Fellowship under Grant No. 2014184874. Any opinion, findings, and conclusions or recommendations expressed in this material are those of the authors and do not necessarily reflect the views of the National Science Foundation. The Center for Exoplanets and Habitable Worlds is supported by the Pennsylvania State University, the Eberly College of Science, and the Pennsylvania Space Grant Consortium. JTW and EBF acknowledge support from multiple NASA Keck PI Data Awards, administered by the NASA Exoplanet Science Institute, to follow multiple exoplanets systems including HD 164922 and HD 143761 from semester 2010A to through 2012B (semester 2010B excluded). JTW acknowledges support from NSF grant AST-1211441 GWH acknowledges support from NASA, NSF, Tennessee State University, and the State of Tennessee through its Centers of Excellence program. This research made use of the Exoplanet Orbit Database and the Exoplanet Data Explorer atexoplanets. org. Based on observations obtained at the Gemini Observatory, which is operated by the Association of Universities for Research in Astronomy, Inc., under a cooperative agreement with the NSF on behalf of the Gemini partnership: the National Science Foundation (United States), the National Research Council (Canada), CONICYT (Chile), Ministerio de Ciencia, Tecnologa e Innovacin Productiva (Argentina), and Ministrio da Cincia, Tecnologia e Inovao (Brazil). This work made use of the SIMBAD database (operated at CDS, Strasbourg, France), and NASA's Astrophysics Data System Bibliographic Services. Finally, the authors wish to extend special thanks to those of Hawai'ian ancestry on whose sacred mountain of Maunakea we are privileged to be guests. Without their generous hospitality, the Keck observations presented herein would not have been possible. Research at the Lick Observatory is partially supported by a generous gift from Google.

Facilities: Automated Planet Finder (Levy), Keck:I (HIRES), CoRoT.

\section{REFERENCES}

Asplund, M., Grevesse, N., Sauval, A. J., \& Scott, P. 2009, ARA\&A, 47, 481 Baglin, A., Auvergne, M., Barge, P., Deleuil, M., Michel, E. \& CoRoT Exoplanet Science Team 2009, in IAU Symp. 253, ed. F. Pont, D. Sasselov, \& M. J. Holman (Cambridge: Cambridge Univ. Press), 71

Baglin, A., Michel, E. \& CoRoT Team 2012, in ASP Conf. Ser. 462, Progress in Solar/Stellar Physics with Helio- and Asteroseismology, ed. H. Shibahashi, M. Takata, \& A. E. Lynas-Gray (San Francisco, CA: ASP), 492

Barban, C., Deheuvels, S., Goupil, M. J., et al. 2013, JPhCS, 440, 012030 Bonneau, D., Clausse, J.-M., Delfosse, X., et al. 2006, A\&A, 456, 789 Bonneau, D., Delfosse, X., Mourard, D., et al. 2011, A\&A, 535, A53 Boyajian, T. S., McAlister, H. A., Baines, E. K., et al. 2008, ApJ, 683, 424 Butler, R. P., Marcy, G. W., Williams, E., et al. 1996, PASP, 108, 500 Butler, R. P., Wright, J. T., Marcy, G. W., et al. 2006, ApJ, 646, 505 Chaplin, W. J., \& Miglio, A. 2013, ARA\&A, 51, 353

Chatterjee, S., \& Tan, J. C. 2014, ApJ, 780, 53
Chiang, E., \& Laughlin, G. 2013, MNRAS, 431, 3444

Christensen-Dalsgaard, J., \& Frandsen, S. 1983, SoPh, 82, 469

Claret, A., \& Bloemen, S. 2011, A\&A, 529, A75

Coelho, P. R. T. 2014, MNRAS, 440, 1027

Cumming, A., Butler, R. P., Marcy, G. W., et al. 2008, PASP, 120, 531

Cutri, R. M., Skrutskie, M. F., van Dyk, S., et al. 2003, yCat, 2246, 0

Demory, B.-O. 2014, ApJL, 789, L20

Dotter, A., Chaboyer, B., Jevremović, D., et al. 2008, ApJS, 178, 89

Dumusque, X., Udry, S., Lovis, C., Santos, N. C., \& Monteiro, M. J. P. F. G. 2011, A\&A, 525, A140

Eastman, J., Gaudi, B. S., \& Agol, E. 2013, PASP, 125, 83

Eaton, J. A., Henry, G. W., \& Fekel, F. C. 2003, in Astrophysics and Space Science Library, Vol. 288, ed. T. D. Oswalt (Dordrecht: Kluwer Academic Publishers), 189

Fabrycky, D. C., Lissauer, J. J., Ragozzine, D., et al. 2014, ApJ, 790, 146

Farrington, C. D., ten Brummelaar, T. A., Mason, B. D., et al. 2010, AJ, 139,2308

Fischer, D. A., Anglada-Escude, G., Arriagada, P., et al. 2016, PASP, 128, 066001

Ford, E. B. 2006, ApJ, 642, 505

Fressin, F., Torres, G., Charbonneau, D., et al. 2013, ApJ, 766, 81 Fuhrmann, K., Pfeiffer, M. J., \& Bernkopf, J. 1998, A\&A, 336, 942

Fulton, B. J., Howard, A. W., Winn, J. N., et al. 2013, ApJ, 772, 80

Fulton, B. J., Weiss, L. M., Sinukoff, E., et al. 2015, ApJ, 805, 175

Gatewood, G., Han, I., \& Black, D. C. 2001, ApJL, 548, L61

Ghezzi, L., Cunha, K., Smith, V. V., et al. 2010, ApJ, 720, 1290

Gladman, B. 1993, Icar, 106, 247

González Hernández, J. I., Israelian, G., Santos, N. C., et al. 2010, ApJ, 720,1592

Gray, R. O., Corbally, C. J., Garrison, R. F., McFadden, M. T., \& Robinson, P. E. 2003, AJ, 126, 2048

Hall, J. C., Henry, G. W., Lockwood, G. W., Skiff, B. A., \& Saar, S. H. 2009, AJ, 138,312

Han, E., Wang, S. X., Wright, J. T., et al. 2014, PASP, 126, 827

Hansen, B. M. S., \& Murray, N. 2012, ApJ, 751, 158

Henry, G. W. 1999, PASP, 111, 845

Henry, G. W., Kane, S. R., Wang, S. X., et al. 2013, ApJ, 768, 155

Horch, E. P., Gomez, S. C., Sherry, W. H., et al. 2011, AJ, 141, 45

Horch, E. P., Howell, S. B., Everett, M. E., \& Ciardi, D. R. 2012, AJ, 144, 165

Horch, E. P., Veillette, D. R., Baena Gallé, R., et al. 2009, AJ, 137, 5057

Howard, A. W., \& Fulton, B. J. 2016, PASP, 128, 114401

Howard, A. W., Johnson, J. A., Marcy, G. W., et al. 2009, ApJ, 696, 75

Howard, A. W., Johnson, J. A., Marcy, G. W., et al. 2010, Sci, 330, 653

Howard, A. W., Johnson, J. A., Marcy, G. W., et al. 2011a, ApJ, 726, 73

Howard, A. W., Johnson, J. A., Marcy, G. W., et al. 2011b, ApJ, 730, 10

Howard, A. W., Marcy, G. W., Bryson, S. T., et al. 2012, ApJS, 201, 15

Howard, A. W., Marcy, G. W., Fischer, D. A., et al. 2014, ApJ, 794, 51

Howell, S. B., Everett, M. E., Sherry, W., Horch, E., \& Ciardi, D. R. 2011, AJ, 142,19

Huber, D., Bedding, T. R., Stello, D., et al. 2011, ApJ, 743, 143

Ida, S., \& Lin, D. N. C. 2004, ApJ, 604, 388

Ida, S., \& Lin, D. N. C. 2008, ApJ, 673, 487

Ireland, M. J., Mérand, A., ten Brummelaar, T. A., et al. 2008, Proc. SPIE, 7013, 24

Isaacson, H., \& Fischer, D. 2010, ApJ, 725, 875

Jenkins, J. M., Chandrasekaran, H., McCauliff, S. D., et al. 2010, Proc. SPIE, 7740, 77400D

Johnson, J. A., Clanton, C., Howard, A. W., et al. 2011, ApJS, 197, 26

Kallinger, T., Weiss, W. W., Barban, C., et al. 2010, A\&A, 509, A77

Kane, S. R., Howell, S. B., Horch, E. P., et al. 2014, ApJ, 785, 93

Kjeldsen, H., \& Bedding, T. R. 1995, A\&A, 293, 87

Knutson, H. A., Fulton, B. J., Montet, B. T., et al. 2014, ApJ, 785, 126

Koen, C., Kilkenny, D., van Wyk, F., \& Marang, F. 2010, MNRAS, 403, 1949

Lee, E. J., \& Chiang, E. 2015, ApJ, 811, 41

Lee, E. J., Chiang, E., \& Ormel, C. W. 2014, ApJ, 797, 95

Lockwood, G. W., Henry, G. W., Hall, J. C., \& Radick, R. R. 2013, in ASP Conf. Ser. 472, New Quests in Stellar Astrophysics III: A Panchromatic View of Solar-Like Stars, With and Without Planets, ed. M. Chavez et al. (San Francisco, CA: ASP), 203

Lomb, N. R. 1976, Ap\&SS, 39, 447

Lopez, E. D. 2014, PhD thesis, Univ. California, http://adsabs.harvard.edu/ abs/2014PhDT.......106L

Lopez, E. D., \& Fortney, J. J. 2014, ApJ, 792, 1

Mann, A. W., \& von Braun, K. 2015, PASP, 127, 102

Mayor, M., Marmier, M., Lovis, C., et al. 2011, arXiv:1109.2497

Medhi, B. J., Messina, S., Parihar, P. S., et al. 2007, A\&A, 469, 713 
Meléndez, J., Asplund, M., Gustafsson, B., \& Yong, D. 2009, ApJL, 704, L66

Michel, E., Baglin, A., Auvergne, M., et al. 2008, Sci, 322, 558

Mordasini, C., Alibert, Y., \& Benz, W. 2009, A\&A, 501, 1139

Morel, T., Rainer, M., Poretti, E., Barban, C., \& Boumier, P. 2013, A\&A, 552, A42

Morton, T. D. 2015, Isochrones: Stellar Model Grid Package, Astrophysics Source Code Library, ascl:1503.010

Motalebi, F., Udry, S., Gillon, M., et al. 2015, A\&A, 584, A72

Noyes, R. W., Jha, S., Korzennik, S. G., et al. 1997, ApJL, 483, L111

O'Toole, S. J., Jones, H. R. A., Tinney, C. G., et al. 2009, ApJ, 701, 1732

Pepe, F., Mayor, M., Queloz, D., et al. 2004, A\&A, 423, 385

Petigura, E. A. 2015, arXiv:1510.03902

Petigura, E. A., Howard, A. W., \& Marcy, G. W. 2013a, PNAS, 110, 19273

Petigura, E. A., Marcy, G. W., \& Howard, A. W. 2013b, ApJ, 770, 69

Pickles, A. J. 1998, PASP, 110, 863

Queloz, D., Henry, G. W., Sivan, J. P., et al. 2001, A\&A, 379, 279

Radovan, M. V., Lanclos, K., Holden, B. P., et al. 2014, Proc. SPIE, 9145, 2

Raghavan, D., Farrington, C. D., ten Brummelaar, T. A., et al. 2012, ApJ, 745,24

Ramírez, I., Fish, J. R., Lambert, D. L., \& Allende Prieto, C. 2012, ApJ, 756, 46

Ramírez, I., Meléndez, J., Bean, J., et al. 2014, A\&A, 572, A48

Reffert, S., \& Quirrenbach, A. 2011, A\&A, 527, A140
Santos, N. C., Sousa, S. G., Mortier, A., et al. 2013, A\&A, 556, A150 Scargle, J. D. 1982, ApJ, 263, 835

Schwarzenberg-Czerny, A. 1998, MNRAS, 301, 831

Spearman, C. 1904, The American Journal of Psychology, 15, 72

Swift, J. J., Bottom, M., Johnson, J. A., et al. 2015, JATIS, 1, 027002

ten Brummelaar, T. A., McAlister, H. A., Ridgway, S. T., et al. 2005, ApJ, 628,453

Ter Braak, C. 2006, Stat. Comput., 16, 239, http://link.springer.com/article/ 10.1007/s11222-006-8769-1

Torres, G., Andersen, J., \& Giménez, A. 2010, A\&ARv, 18, 67

Valenti, J. A., \& Fischer, D. A. 2005, ApJS, 159, 141

van Belle, G. T., \& von Braun, K. 2009, ApJ, 694, 1085

van Leeuwen, F. 2007, A\&A, 474, 653

Veras, D., \& Ford, E. B. 2012, MNRAS, 420, L23

Vogt, S. S., Allen, S. L., Bigelow, B. C., et al. 1994, Proc. SPIE, 2198, 362

Vogt, S. S., Radovan, M., Kibrick, R., et al. 2014, PASP, 126, 359

von Braun, K., Boyajian, T. S., van Belle, G. T., et al. 2014, MNRAS, 438, 2413

Weiss, L. M., \& Marcy, G. W. 2014, ApJL, 783, L6

White, T. R., Huber, D., Maestro, V., et al. 2013, MNRAS, 433, 1262

Wright, J. T., \& Eastman, J. D. 2014, PASP, 126, 838

Wright, J. T., Marcy, G. W., Fischer, D. A., et al. 2007, ApJ, 657, 533

Zucker, S., \& Mazeh, T. 2001, arXiv:astro-ph/0104098 